\title{
NBS
}

Eechnical Note

no. 301

\section{ON THE FORMULATION AND NUMERICAL EVALUATION OF A SET OF TWO-PHASE FLOW EQUATIONS MODELLING THE COOLDOWN PROCESS}

Stephen Jarvis, Jr.

U. S. DEPARTMENT OF COMMERCE NATIONAL BUREAU OF STANDARDS 


\section{THE NATIONAL BUREAU OF STANDARDS}

The National Bureau of Standards is a principal focal point in the Federal Government for assuring maximum application of the physical and engineering sciences to the advancement of technology in industry and commerce. Its responsibilities include development and maintenance of the national standards of measurement, and the provisions of means for making measurements consistent with those standards; determination of physical constants and properties of materials; development of methods for testing materials, mechanisms, and structures, and making such tests as may be necessary, particularly for government agencies; cooperation in the establishment of standard practices for incorporation in codes and specifications; advisory service to government agencies on scientific and technical problems; invention and development of devices to serve special needs of the Government; assistance to industry, business, and consumers in the development and acceptance of commercial standards and simplified trade practice recommendations; administration of programs in cooperation with United States business groups and standards organizations for the development of international standards of practice; and maintenance of a clearinghouse for the collection and dissemination of scientific, technical, and engineering information. The scope of the Bureau's activities is suggested in the following listing of its four Institutes and their organizational units.

Institute for Basic Standards. Electricity. Metrology. Heat. Radiation Physics. Mechanics. Applied Mathematics. Atomic Physics. Physical Chemistry. Laboratory Astrophysics.* Radio Standards Laboratory: Radio Standards Physics; Radio Standards Engineering.** Office of Standard Reference Data.

Institute for Materials Research. Analytical Chemistry. Polymers. Metallurgy. Inorganic Materials. Reactor Radiations. Cryogenics. ** Office of Standard Reference Materials.

Central Radio Propagation Laboratory.** Ionosphere Research and Propagation. Troposphere and Space Telecommunications. Radio Systems. Upper Atmosphere and Space Physics.

Institute for Applied Teclinology. Textiles and Apparel Technology Center. Building Research. Industrial Equipment. Information Technology. Performance Test Development. Instrumentation. Transport Systems. Office of Technical Services. Office of Weights and Measures. Office of Engineering Standards. Office of Industrial Services.

* NBS Group, Joint Institute for Laboratory Astrophysics at the University of Colorado.

** Located at Boulder, Colorado. 


\title{
NATIONAL BUREAU OF STANDARDS Eechnical Mote 301 \\ Issued January 4, 1965
}

\section{ON THE FORMULATION AND NUMERICAL EVALUATION OF A SET OF TWO.PHASE FLOW EQUATIONS MODELLING THE COOLDOWN PROCESS}

\author{
Stephen Jarvis, Jr. \\ National Bureau of Standards \\ Boulder, Colorado
}

NBS Technical Notes are designed to supplement the Bureau's regular publications program. They provide a means for making available scientific data that are of transient or limited interest. Technical Notes may be listed or referred to in the open literature.

\footnotetext{
For sale by the Superlntendent of Documents, U. S. Government Printing Offlce

Washlnqtor. D.C. 20402

Price: 55 cents
} 



\section{CONTENTS}

Page

Abstract-1-0.-1

1. Introduction-_...- I

2. Basic Equations (2V2T)

3. The Reduced System (IV2T) - 10

4. Auxiliary Routines-_-...- 23

5. Controls and Blocks-a 29

6. Computation Summary-1

7. Glossary--1-1- 36

8. References-_. 40

Appendix - The Finite Difference Method of Lax-_-_-_-_-_-_ 41

Figure 1--0.-1 4 ?

Figure 2-1 43

Figure 3-1 44

Figure 4-0.-n 45

Figure 5-1. 46

Figure 6-1 47

Figure 7 -and 48 

On the Formulation and Numerical Evaluation of a Set of

Two-Phase Flow Equations Modelling the Cooldown Process

Stephen Jarvis, Jr.

A model of transient two-phase flow in a pipe is constructed in Eulerian coordinates assuming a single velocity but independent temperatures in the two phases. Experiments on the numerical integration of the system for Cooldown problem by both Iax and Courant-Isaacson-Rees methods indicate that a very fine spatial difference net must be used to compensate for the numerical diffusion essential to computational stability if a second surge is to be realized.

\section{Introduction}

This report is intended to summarize progress to date on the formulation and numerical integration of systems of equations modelling general two-phase flows in a pipe. Principal application would be to the study of the development of choked flows in tubes of varying cross-section, and to the cooldown problem. The latter problem, the cooling of warm transfer lines resulting from the flow of cryogenic liquids, has been the target of the present study.

In a private communication, a set of Cooldown equations was proposed based on conservation laws for a single fluid, and three distinct phases were admitted: all gas, all (compressible) liquid, and a two-phase medium based on a quality weighted average of gas and liquid at a single velocity $(V)$, pressure $(p)$, and temperature $(T)$. State changes in the two-phase domain were confined to the vapor-pressure curve with quality (y) satisfying $0<y<1$. The complicated thermodynamic structure of the fluid $\left(\mathrm{N}_{2}\right)$ was approximated by least squares fit to empirical curves. The numerical approach taken, Picone's method, involved polynomial approxImation in $x$ and $t$ using the governing equations to determine coefficients. This method is not well suited, however, to hyperbolic systems of partial differential equations (PDE's) whose solutions may have discontinuous derivatives at interior points (and in the case of non-linear equations, even discontinuous variables, or shocks), although it is applicable to elliptic and parabolic systems for which a high degree of smoothness in the solutions is the rule. Attempts to force polynomial fits to 
discontinuities results in highly unstable behavior, which was, indeed, observed. Finite difference schemes are generally more successful with hyperbolic systems.

In addition to the restrictive assumption of homogeneity on the twophase medium in the above model the location of phase boundaries is a serious problem. If $\Delta x$ is the spatial difference interval, it is useless to attempt to resolve phase domains on a scale even as small as $\Delta x$. A reasonable value of $\Delta x$, based on computing time, is 10 feet for the Cooldown problem, so that it is clear that if the flow is not insensitive to the structure of phase domains of this scale, the problem is computationally impossible. Clearly the small scale spontaneous generation and degeneration of two-phase pockets is beyond the scope of the numerical approach in general, and broad scale averages are at all times under consideration.

A fundamental property of continuous hyperbolic systems is that the flow at a point $(x, t)$ is completely determined by the flow at time $t-\Delta t$ on the interval $(x-\lambda \Delta t, x+\lambda \Delta t)$ (where $\Delta t$ is small), the so-called "domain of dependence". Here $\lambda$ is the highest signal speed in the fluid at $(x, t)$. For given $\Delta x$ then, the smaller is $\lambda$, the shorter is the computation time required to integrate over a given real time interval. Since liquids in general have very high signal speeds, it is obvious that compressibility will be a major factor in the computing economics. Furthermore, few two-phase problems of interests will involve "hammer" (liquid-shock), so that considering the liquid to be incompressible would be both reasonable and economical. A further simplification that can be made which will probably not be more significant than those already discussed is to assume the gas to be a perfect gas and approximate the vapor-pressure curve by a straight line to reduce computation.

The flow of incompressible liquid (having infinite signal velocity) can only be handled in the finite difference scheme by considering the dynamics of complete liquid columns bounded at each end by compressible media. Thus the problem of scale length and phase boundaries again appears. 
Since we are in any case restricted to the consideration of twophase flows which do not depend critically on "phase resolution," it appears that the whole matter could be greatly simplified by allowing only two-phase flow everywhere, $0<y_{\ell} \leqslant y \leqslant y_{4}<1$, placing then a minimum and a maximum on the quality $y(x, t)$. A small volume of gas then, while storing insignificant quantities of energy and momentum, adds compressibility to the "all-liquid" flow, $y=y_{\ell}$. A sufficiently small quantity of liquid can similarly have no effect on the "all-gas" flow, $y=y_{u}$.

Taking this approach, we must generalize our models now to admit both a liquid and a gas temperature to avoid being confined to the vapor pressure curve. This extension appears to be a worthwhile generalization in that heat transfer between gas and liquid is controllable, while supercooling and super-heating are admissible. A system of five first-order partial differential equations (system "IV2T") is needed to describe the flow, involving a single velocity $v(x, t)$, pressure $p(x, t)$, gas density $r(x, t)$, gas temperatures $T_{g}(x, t)$, and liquid temperatures $T_{\ell}(x, t)$. (Iongitudinal heat conduction in the wall is ignored also, so that the wall temperature is controlled by a single first-order equation in time coupled to the five mentioned above only through source terms and is of no mathematical consequence).

For further generality in a one-dimensional model a 6 th equation was added (forming system "2V2T") to allow both a gas velocity $V(x, t)$ and a liquid velocity $U(x, t)$, and requiring a description of momentum coupling between the two fluids. But unless the difference $|U-V|$ is adequately restricted (by friction, for example), the initial value problem for this system is not "well-posed", i.e., there are fundamental hydrodynamic stability problems involved. (This is evidenced by complex characteristics). This 6th order system was dropped, then, in favor of the 5th order (one velocity) system pending a study of the meaning of this instability.

In any fluid system, two natural coordinate systems are available: the system fixed in space (fixed in the pipe) is called the Eulerian 
system, while a system fixed in the fluid is called Lagrangian. Only in one-dimensional transient flows does the Lagrangian approach offer some advantages over the Eulerian, although the fixed boundary conditions (at the pipe ends) of the Eulerian scheme go over into variable boundary conditions. From the coding point of view, the finite difference method in Lagrangian coordinates has the great disadvantage that the difference net shifts in and out of the pipe and may get too dense or too sparse to admit good approximation to the dynamical equations. There is involved frequent interpolation and renumbering of net points. Furthermore, in the (2V2T) system, the occurrence of two velocities complicates the Iagrangian system, (and might eliminate the natural advantage of the Lagrangian system).

On the other hand, the Iagrangian schemes would have minimized the difficulty caused by the great variations in density and momentum density that the two phase system presents, and might have described the movement of "liquid points" without the large mass diffusion which turns out to be the most serious problem of the Eulerian approach.

Experimentation with a Lagrangian formulation would be very desirable. It would involve no new principles, but some tedious coding difficulties .

\section{Basic Equations (2V2T)}

We shall assume the pipe has a variable cross-sectional area $A(x)$ shared between a gas stream of cross-sectional area $\mathrm{zA}$ and an incompressible liquid stream of cross-sectional area $(l-z) A$, so that $z(x, t)$ is a volumetric quality.

Iet $\mathrm{s}$ be the constant liquid density, and $\mathrm{E}$ and $\mathrm{F}$ be specific energies of gas and liquid.

In terms of source strength/unit length, $S_{j}$, the conservation laws are readily written for the two streams.

Liquid Continuity,

$$
(A(1-z) s)_{, t}+\left(A(1-z) s(1)_{x}=S_{1}\right.
$$


Gas Continuity,

$$
(A z r)_{t}+(A z r V)_{x}=S_{2}
$$

Liquid Momentum,

$$
\begin{array}{r}
(A(1-z) s \cup)_{, t}+\left(A(1-z) s U^{2}+A(1-z) p\right)_{x x} \\
-p((1-z) A)_{, x}=S_{31}
\end{array}
$$

Gas Momentum,

$$
\begin{aligned}
(A z r V)_{, t}+ & \left(A z r V^{2}+A_{z p}\right)_{-x} \\
& -p(z A)_{x}=S_{32}
\end{aligned}
$$

Liquid Energy,

$$
\begin{aligned}
& {\left[A(1-z)_{s}\left(\frac{1}{2} U^{2}+F\right)\right]_{t}} \\
& \quad+\left[A(1-z)_{s} U\left(\frac{1}{2} U^{2}+F\right)+A(1-z) U p\right]_{x}=S_{s}
\end{aligned}
$$

Gas Energy,

$$
\begin{aligned}
& {\left[A z r\left(\frac{1}{2} V^{2}+E\right)\right]_{t}} \\
& \quad+\left[A z r V\left(\frac{1}{2} V^{2}+E\right)+A z V p\right]_{x}=S_{4}
\end{aligned}
$$

Wall Temperature,

$$
T_{w, t}=\delta_{c}
$$

We assume a state equation for the gas,

$$
p=P(r, E)
$$

5 
Calling $e$ and $c$ the mass rates of evaporation and condensation per unit length of pipe at $(x, t)$, we have

$$
S_{1}=c-e
$$

and

$$
S_{2}=e-c
$$

Let $l_{9}, l_{l}, l_{0}$ be perimeters of contact between gas-wall, liquidwall, and gas-liquid, respectively, at the section $(x, t)$, and $\tau_{g}, \tau_{\ell}, \tau_{0}$ the associated shear stresses (wall on gas, wall on liquid, liquid on gas). Assume gravitational acceleration $g(x)$ in positive - $x$ direction given. The $q_{g}$ and $q_{\ell}$ are Won Newman - Richtmyer nonlinear pseudo-viscous forces introduced to permit shocks, [2] and are described later. Then we set:

$$
\begin{aligned}
S_{31}=A s(1-z) g & -\tau_{l} l_{l}+\tau_{0} l_{0} \\
& -e V+c U-A(1-z) g_{\ell, x}
\end{aligned}
$$

and

$$
\begin{array}{rl}
S_{32}=A r z & g-\tau_{g} l_{g}-\tau_{0} l_{0} \\
& +\epsilon V-c U-A z q g, x
\end{array}
$$

Let $q_{q}, q_{0}$ be heat transfer rate from wall-into-gas and liquid-intogas per unit area, and $q_{\ell}$ the wall-liquid rate. Then:

$$
\begin{aligned}
S_{s} & =(1-z) s \cup g-q_{0} l_{0}+q_{l} l_{l} \\
& -l\left(\frac{1}{2} V^{2}+E+\frac{1}{r} p\right)+c\left(\frac{1}{2} U^{2}+F+\frac{1}{s} p\right) \\
& -\left(A(1-z) \cup q_{l}\right)_{x}
\end{aligned}
$$

and

$$
\begin{aligned}
S_{4}= & z r V g A+q_{0} l_{0}+q_{g} l_{g} \\
& +e\left(\frac{1}{2} V^{2}+E+\frac{1}{r} p\right)-c\left(\frac{1}{2} U^{2}+F+\frac{1}{s} p\right) \\
& -\left(A z V_{g}\right)_{x}
\end{aligned}
$$

6 
We have taken the following point of view in formulating these sources. When a quantity of liquid $e \Delta t$ is evaporated, its specific momentum and specific enthalpy are brought to those values in the gas stream at the expense of the liquid stream, and contrariwise for condensation. For example, the gas momentum source term is e $V-c U$ rather than $e U-c V$. (In a continuous three-dimensional model, the phase change takes place on an interface on which $U=V, T_{g}=T_{l}$. The option here arises from the fact that in the one-dimensional model, the dependent variables are averages over portions of stream cross-sections.) This has worked out better than the alternative because the mass $e \Delta t$ is normally taken to be due to some part of the energy transferred into the liquid in time $\Delta t$. Hence, in a small finite interval $\Delta t$, there is less danger of removing more energy from a stream than is available. Thus,

$$
S_{6}=-h_{w x}\left(q_{\ell} \ell_{b}+q_{q} \ell_{g}\right) / A
$$

A considerable effort was expended in integrating this system by the Iax method ${ }^{[3]}$ (Appendix) although it fails to fit the model of "Conservation Form" by the presence of the term $-\mathrm{p}(\mathrm{Az})$, $_{\mathrm{x}}$ in (4) and the analogous term in (5). The instability which plagued this approach was probably not due to this fact, however, but was due to the fact the system may become non-hyperbolic, i.e., it may have complex-valued signal speeds, as is shown below. Since the Iax finite difference form is subject to the "domain of dependence" restrictions associated with the maximum signal speed in the medium, a calculation of signal speeds was made. By expanding the (2V2T) system to a quasi-linear system of the form

$$
a_{i j} u_{g, t}+b_{i j} u_{j, x}=S_{i}
$$

the signal speeds $\lambda$ are found as solutions of the system: [2]

$$
\operatorname{det}\left|b_{i j}-\lambda a_{i j}\right|=0
$$


Following is a 6 th order algebraic equation in $\lambda$ whose coefficients are functions of the flow variables (but not their derivatives):

$$
\begin{gathered}
o=(\lambda-U)(\lambda-V)\left\{(\lambda-U)^{2}\left[\mu-v-(\lambda-V)^{2}\right]\right. \\
-\omega(\lambda-V)[\mu V+\nu(\lambda-V)]
\end{gathered}
$$

where

$$
\begin{array}{ll}
\omega=\frac{r(1-z)}{s z} & (\omega>0) \\
\mu=\frac{p}{r^{2}} \frac{\partial p}{\partial E} & (\mu>0) \\
\nu=-\frac{\partial p}{\partial r} & (v<0)
\end{array}
$$

In general, $\omega$ will be small, since $z \geqslant .05$ will be taken, and will vanish in the case of all gas where $z=1$. When $\omega$ is small, and $U \neq V$, we find the roots

$$
\lambda=\left\{\begin{array}{l}
V \\
U \\
U+\omega^{1 / 2} \phi+\theta(\omega) \\
U-\omega^{1 / 2} \phi+\theta(\omega) \\
V+\sqrt{\mu-\nu}+\theta(\omega) \\
V-\sqrt{\mu-\nu}+\theta(\omega)
\end{array}\right.
$$

where

$$
\phi=\left[\frac{(U-V)((u-v) V+\nu U)}{(u-v)-(U-V)^{2}}\right]^{1 / 2}
$$

8 
Then as

$$
\begin{aligned}
& U \rightarrow V, \\
& \phi \rightarrow\left[\left(\frac{\mu}{\mu-v}\right) V(U-V)\right]^{1 / 2}
\end{aligned}
$$

which is imaginary for

$$
V(U-V)<0
$$

For large $\omega$,

$$
\lambda=\left\{\begin{array}{l}
V \\
u \\
V \\
V(1-\mu / v) \\
\pm(-\omega v)^{1 / 2}
\end{array}\right.
$$

On the other hand, if there is sufficient friction between the streams so that $|v-U|$ is not large at interior points of the $(x, t)$ domain (as must be expected for small $\omega$ ), the above limits do not apply. Let us assume the friction is large enough that

$$
U=V+\omega \omega
$$

where $v$ is bounded as $\omega \rightarrow 0$. We obtain the roots:

$$
\lambda=\left\{\begin{array}{l}
V \\
U \\
V \pm \sqrt{\mu-v}+\theta(\omega) \\
U+\omega \frac{\mu V}{2(\mu-v)}\left(1 \pm \sqrt{1+4 \frac{\mu-v}{v}}\right)+\theta\left(\omega^{2}\right)
\end{array}\right.
$$

which are all real for small $\omega$ at least. No attempt was made to determine how stringent is the restriction on the interface friction and initial values of the functions imposed by the requirement of real roots, and a study of this (2V2T)-system was postponed in favor of the 
(IV2T)-system. (Improperly posed initial value problems, as would be one with complex roots, occur in unstable problems, as for example the flow following the collision of two liquid masses).

3. The Reduced System (I V2T)

Henceforth, we shall discuss only the system with a single velocity V obtained by adding (3) and (4) and setting $U=V$ throughout. We obtain an equation for the conservation of total momentum which is

$$
\begin{aligned}
& (A p V)_{, t}+\left(A \rho V^{2}+A p\right)_{x}-p A_{x}=\int_{3} \\
& p \equiv r z+s(1-z) \\
& S_{3} \equiv A \rho g-\tau_{l} l_{l}-\tau_{g} l_{g} \\
& -A(1-z) q_{e, x}-A_{z q} q_{9, x}=S_{3 !}+S_{32}
\end{aligned}
$$

The resulting system (IV2T) ${ }_{A}$, is entirely in "Conservation Form" (A, ${ }_{X}$ a given function: see Appendix) and was programmed in the Lax scheme. Difficulties are encountered when area variation occurs due to the structure of the Lax Derivatives. An alternate $\left(\right.$ IVZT) ${ }_{B}$ system, also in "Conservation Form" is obtained by removing $A(x)$ from the time derivative.

Let $B(x)=1 / A(x)$; then

$$
\begin{gathered}
Z(x)=A_{x} / A=-B, x / B \\
z_{, t}-((1-z) V)_{x}-(1-z) V Z=-S_{1} B / s \\
(r z)_{, t}+(r z V)_{x}+r z V Z=\int_{2} B
\end{gathered}
$$

10 


$$
\begin{aligned}
& (\rho V)_{t}+\left(\rho V^{2}+p\right)_{x}+\rho V^{2} Z=J_{3} B \\
& \left(r z\left(\frac{1}{2} V^{2}+E\right)\right)_{t}+\left(r z V\left(\frac{1}{2} V^{2}+E+\frac{1}{r} p\right)\right)_{x} \\
& +r z\left(\frac{1}{2} V^{2}+E+\frac{1}{r} p\right) V Z=J_{+} B \\
& \left((1-z)\left(\frac{1}{2} V^{2}+F\right)\right)_{t}+\left((1-z) V\left(\frac{1}{2} V^{2}+F+\frac{1}{s} p\right)\right)_{x} \\
& +(1-z)\left(\frac{1}{2} V^{2}+F+\frac{1}{s} p\right) V Z=J_{5} B
\end{aligned}
$$

This was programmed in the Iax scheme (using $q_{g}=q_{b}=0$ ). The result was entirely unsatisfactory due to the essential averaging process implicit in the system. It appeared that the CIR (Courant-Isaacson-Rees) ${ }^{[1]}$ method would avoid much of this spreading, and could yet handle shocks by inclusion of the concentrated pseudo-viscous diffusion $\left(q_{g}, q_{l}\right)$. These terms must be considered as source terms, however, and not a part of the signalgenerating structure basic to the CIR methods. The presence of the diffusion terms required the inclusion of an upper bound on the quantity $\Delta t /(\Delta x)^{2}$ based on stability in parabolic systems. [3] only when strong shocks are present is this term comparable to the maximum signal speed limit. The term used was

$$
\Delta t \leqslant \begin{cases}\min _{\{x\}}\left(\frac{\rho \Delta x}{32 r|V(x+\Delta x)-V(x)|}\right) & \left\{\begin{array}{c}
\text { shock diffusion } \\
\text { condition }
\end{array}\right\} \\
\min _{\{x\},\{\lambda\}}\left(\frac{\Delta x}{|\lambda|}\right) & \left\{\begin{array}{c}
\text { domain of dependence } \\
\text { condition }
\end{array}\right\}\end{cases}
$$


The equations treated by the CIR method are the quasi-linear forms derived from $(I \mathrm{~V} 2 \mathrm{~T})_{\mathrm{B}}$, and called $(\mathrm{IV} 2 \mathrm{~T})_{\mathrm{C}}$ :

$$
\begin{aligned}
& z_{, t}+V_{z, x}^{\prime}-(1-z) V_{, x}=D_{1} \\
& r_{t}+V_{r_{x}}+\frac{r}{z} V_{x}=D_{2} \\
& V_{, t}+V V_{, x}+\frac{1}{\rho}\left(P_{, E} E_{, x}+P_{r} r_{x}\right)=D_{3} \\
& E_{, t}+V E_{, x}+\frac{(s-r)(1-z)}{r \rho} V\left(P_{, E} E_{j x}+P_{\mu_{r}} r_{x x}\right) \\
& +\frac{V p}{r z} z_{x}+\frac{p}{r} V_{x}=D_{+} \\
& F_{, t}+V F_{, x}-\frac{(s-r) z}{s p} V\left(P_{, E} E_{x}+P_{r_{r}} F_{x}\right) \\
& +\frac{p}{s} V_{x}-\frac{p V}{s(1-z) z_{x x}}=D_{s}
\end{aligned}
$$

where for convenience we have broken the sources into the following forms :

$$
D_{i}=\hat{D}_{i 1}+D_{i 2}(e B)+D_{i 3}(e B)
$$

12 


$$
\begin{aligned}
& \hat{D}_{11}=V Z(1-z) \\
& D_{12}=-D_{13}=\frac{1}{5} \\
& \hat{D}_{21}=-\frac{r V}{z} Z \\
& D_{22}=-D_{23}=-\frac{s-r}{s z} \\
& \hat{D}_{31}=g-\frac{1}{\rho}\left(\tau_{g} K_{g}+\tau_{l} K_{l}\right)+\phi_{3} \\
& D_{32}=D_{33}=0 \\
& \hat{D}_{41}=\frac{1}{r z}\left(q_{0} k_{0}+q_{g} k_{9}-\delta_{4}-p V_{z} Z\right) \\
& +\frac{V}{p}\left(\tau_{g} k_{9}+\tau_{l} k_{l}+\phi_{3}\right) \\
& D_{+2}=\left(E-F-\frac{1}{s} p\right) / r z \\
& D_{+3}=\frac{p}{r^{2} z} \\
& \hat{D}_{s 1}=\frac{1}{s}\left(\frac{1}{1-z}\left(q_{l} k_{l}-q_{0} k_{0}\right)-\delta_{s}-p \vee z\right) \\
& +\frac{s V}{p}\left(\tau_{9} k_{9}+\tau_{l} k_{l}+\delta_{3}\right) \\
& D_{52}=\frac{p}{s^{2}(1-z)} \\
& D_{53}=\frac{1}{s(1-z)}\left(F-E-\frac{1}{r} p\right)
\end{aligned}
$$


where

$$
\left\{\begin{array}{l}
k_{0}=B l_{0} \\
k_{g}=B l_{g} \\
k_{l}=B l_{l}
\end{array}\right.
$$

$$
\left\{\begin{array}{l}
f_{3}=\left(z q_{9}\right)_{x}+\left((1-z) q_{l}\right)_{x}+\left(z q_{9}+(1-z) q_{l}\right) z \\
\delta_{4}=\left(z V q_{9}\right)_{x}+z V q_{9} z \\
\delta_{5}=\left[\left((1-z) V q_{l}\right)_{,}+(1-z) V q_{l} z\right] /(1-z)
\end{array}\right.
$$

$$
\begin{aligned}
& q_{g}= \begin{cases}a^{2} r(\Delta x)^{2}\left(\frac{\partial V}{\partial x}\right)^{2} & \left(\frac{\partial V}{\partial x}<0\right) \\
0 & \left(\frac{\partial V}{\partial x} \geqslant 0\right)\end{cases} \\
& q_{l}= \begin{cases}a^{2} s(\Delta x)^{2}\left(\frac{\partial V}{\partial x}\right)^{2} & \left(\frac{\partial V}{\partial x}<0\right) \\
0 & \left(\frac{\partial V}{\partial x} \geqslant 0\right)\end{cases}
\end{aligned}
$$

where $a^{2}$ is a number between $I$ and 2 (In the case of air at standard conditions, this has the effect of spreading steady shocks of moderate strength over three to four net intervals).

14 
This system is of the form

$$
u_{, t}^{i}+b^{i j} u_{, x}^{j}=D_{i} \quad(i=1, \ldots, 5)
$$

(summed on repeated index) with

$$
\begin{aligned}
& \left(b^{i j}\right)=\left(\begin{array}{ccccc}
V & 0 & c^{13} & 0 & 0 \\
0 & V & c^{23} & 0 & 0 \\
0 & c^{32} & V & C^{34} & 0 \\
V c^{41} & c^{42} & C^{43} & C^{44} & 0 \\
c^{51} & c^{52} & c^{53} & c^{54} & V
\end{array}\right) \\
& \left\{\begin{array}{l}
C^{13}=-(1-z) \\
C^{23}=r / z \\
C^{32}=-v / \rho \\
C^{34}=-\mu r / v \rho
\end{array}\right. \\
& C^{41}=-v / z \\
& C^{42}=\left(\frac{s}{r}-1\right) \vee C^{32} /(1-z) \\
& C^{s \prime}=-V p /(s(1-z)) \\
& C^{52}=-\left(1-\frac{r}{5}\right) V_{z} C^{32} \\
& C^{53}=p / s \\
& C^{54}=-\left(1-\frac{r}{s}\right) V_{z} C^{34}
\end{aligned}
$$

15 
We shall assume the perfect gas thermodynamics, which gives

$$
\nu=-\frac{p}{r}, \mu=-(r-i) \nu
$$

where $\gamma$ is the ratio of specific heats. The signal velocities now satisfy the 5 th order system:

$$
\left[\begin{array}{l}
(\lambda-V)^{2}=0 \\
(\lambda-V)^{3}+\frac{\mu V(r-r)(1-z)}{v \rho}(\lambda-V)^{2} \\
-\frac{r(z \mu-v)}{\rho z}(\lambda-V)+\frac{r(1-z) \mu}{\rho z} V=0
\end{array}\right.
$$

While it has not been proved that the cubic has three real roots for all realizable values of the flow variables, these roots were computed from the explicit form for cubics, and no complex roots were encountered. (the program stops in the event of complex roots). By Descartes' Rule of Signs, it is readily proved that, if all 5 roots are real, there are at least 4 roots $\left(\lambda^{(1)}, \ldots, \lambda^{(4)}\right)$ having the same sign as $V$, and at most 1 , $\lambda^{(5)}$, with the same sign as $(-V)$. In the case of "supersonic" flow, all $\lambda^{j}$ have the same sign as $V$. As $V \rightarrow \infty$, only the first two of the cubic terms persist:

$$
(\lambda-V)^{4}=0 \quad \text {, and }(\lambda-V)=\frac{\mu(s-r)(1-z)}{\rho / v /} V
$$

The cases $z \rightarrow \mid$ lead to the pure gas forms,

$$
\lambda^{(3)}=V
$$

and

$$
\lambda^{(4)}, \lambda^{(5)}=V \pm \sqrt{\mu+|\nu|}
$$

16 
while the near-liquid case $z \rightarrow 0$ gives

and

$$
\lambda^{(3)}=\frac{\mu+|v|}{|v|} V
$$

$$
\lambda^{(t)}, \lambda^{(\sigma)}=V \pm \sqrt{\frac{\Gamma(\nu)}{\rho z}}+\theta\left(z^{1 / 2}\right)
$$

gịving infinite signal speeds as $z \rightarrow 0$. However, we shall in practice take $\mathrm{z} \geq \mathrm{z} \min =0.05$. Then

$$
\begin{aligned}
\frac{r|v|}{\rho z} \cong & \left(\frac{r}{s z} \frac{|\nu|}{\mu+|v|}\right)(\mu+|v|) \\
& \leqslant 20 \frac{r}{\gamma s}(\mu+|v|) \\
& <\mu+|v|
\end{aligned}
$$

giving a lower signal speed than pure gas alone (since $\frac{\gamma s}{r}>20$, normally). Thus with a reasonable restriction on $\mathrm{z}, \mathrm{z} \geq 0.05$, the limiting signal velocity will normally be that in the gas.

If all the eigenvalues (signal speeds) $\lambda^{(p)}$ of

$$
\left|\lambda^{(p)} \delta^{i j}-b^{i j}\right|=0
$$

are real, we assume there exists a non-singular transformation satisfying

$$
z^{(p) i}\left(b^{i j}-\lambda^{(p)} \delta^{i j}\right)=0
$$

This has not been proved rigorously, but the following argument makes it a reasonable assumption for this physical system. If the $4 \times 4$ matrix obtained from (58) by deleting row 2 and column 5 is non-singular, then of course the $5 \times 5$ matrix (58) will also be non-singular. This is related to the fact that equations $(32-35)$ of $(1 \mathrm{~V} 2 \mathrm{~T})_{\mathrm{C}}$ do not depend on $F$. For the $4 \times 4$ system, however, a non-singular $z^{i j}$ exists in general when the eigenvalues are distinct, which is "almost always." A small 
independent change in $V$ could get one out of the special case, however, yet cannot be expected to change the mathematical structure of the system. Equation 51 defines a set of left eigenvectors $z^{(p) i}$ corresponding to $\lambda^{(p)},(p=1, \ldots, 5)$. Then applying this transformation to $(28)$, we get

$$
z^{p i} u_{, t}^{i}+z^{p i} b^{i j} u_{, x}^{j}=D^{i} z^{p i}
$$

or

$$
z^{p i}\left(u_{, t}^{i}+\lambda^{(p)} u_{, x}^{i}\right)=D^{i} z^{p i}
$$

(52)

This is the so-called canonical form for hyperbolic systems ${ }^{[3]}$, in which each equation (index $p$ ) has derivatives in a single direction in the $(x, t)$ plane defined by the "characteristic lines" $\Gamma^{(\rho)}$ :

$$
\frac{d x}{d v_{p}}=\lambda^{(p)} \frac{d t}{d v_{p}}
$$

where $\nu_{p}$ is a parameter along $\Pi^{(\rho)}$.

Then

$$
\frac{u_{, \nu_{p}}}{t_{\nu_{p}}}=u_{t}+\lambda^{(p)} u_{x}
$$

so that

$$
{\frac{z^{p i}}{t_{\nu_{p}}}}_{u_{, \nu_{p}}^{i}}=z^{p i} D^{i} \quad(p=1, \ldots, 5)
$$

is, for each $\mathrm{p}$, an ordinary differential equation.

It is in the canonical form (43) (using (45)) that the CIR finite difference scheme is analyzed. When the coefficients and initial data satisfy certain smoothness conditions, then as $\Delta x \rightarrow 0$, the finite ifference scheme is:

$$
\begin{aligned}
& \sum_{i} z^{p i}\left[\frac{u^{i}(x, t+\Delta t)-u^{i}(x, t)}{\Delta t}\right] \\
& +\sum_{i} z^{p^{i}} \lambda^{(p)}\left[ \pm \frac{u^{i}(x+\Delta x, t)-u^{i}(x, t)}{\Delta x}\right]=\sum_{i} D^{i} z^{p^{i}}
\end{aligned}
$$

18 
where $\Delta t \leqslant \operatorname{men}_{\{x, \lambda\}}\left\{\frac{\Delta x}{|\lambda|}\right\}$ and one uses $( \pm)$ according to

$\lambda^{(P)}=\left\{\begin{array}{l}\leqslant 0 \\ >0\end{array} ; \quad\right.$ is stable and converges to a solution of (43) in

some finite, $\Delta x$-independent time interval $\Delta \mathrm{T}>0$ of the initial line. While theoretically valuable, the analysis does not give useful estimates of $\triangle T$ or the error for $\Delta x$. Certainly the procedures could not be continued beyond the formation of the first discontinuity, and might fail before that. The addition of the non-linear pseudo-viscous terms (41-42) has in practice permitted continuation beyond the formation of shocks, but estimates of the error must be inferred from knowledge of the correct solution or experimentation with different values of $\Delta x$. Because of the large number of arithmetic operations involved, a minimum $\Delta \mathrm{x}(\triangle \mathrm{T})$ exists for a given digital word length below which accumulated round-off errors exceed the truncation error. For smaller values of $\Delta x$, all significance in the result will be lost. In the problem at hand, however, it appears that, with the 8-digit word available, the computing time involved for small $\triangle \mathrm{x}$ is a more stringent restriction by far than the round-off error. Taking $Z^{r s}$ to be the inverse of $z^{i j}$,

$$
z^{i j} z^{j s}=z^{j i} Z^{s j}=\delta^{i s}
$$

and we return the system (52) to a form where each equation gives a single time derivative:

$$
u_{, t}^{i}+\sum_{j, p} z^{k_{p}} z^{p j} \lambda^{(p)} u_{, x}^{(p) j}=D^{i}
$$

where the summation is explicitly shown because of the multiple occurrence of the index $p$. The index $p$ on $\stackrel{(p)}{u_{j}}, x$ is to indicate that, in a finite difference scheme according to (54), one should use right- or left-hand difference approximations according as $\lambda^{(P)}$ is negative or positive. 
Since

$$
\sum_{p} z^{r p}\left[z^{p j}\left(b^{j k}-\lambda^{(p)} \delta^{j k}\right)\right] Z^{k s}=0
$$

by (51), using (55) we obtain

$$
\left(b^{r k}-\lambda^{(s)} \delta^{r k}\right) Z^{k s}=0 \text {, }
$$

so that $Z^{k(s)}$. is a right eigenvector corresponding to the eigenvalue $\lambda^{(s)}, s=(1, \ldots, 5)$.

As $V \rightarrow 0$, there is exactly one root, $\lambda^{(3)}$, of the cubic (47) which vanishes. Write

$$
\lambda^{(3)}=\Lambda^{(3)} V
$$

then explicitly, we may choose:

$$
\begin{aligned}
& z^{(i) j}=
\end{aligned}
$$

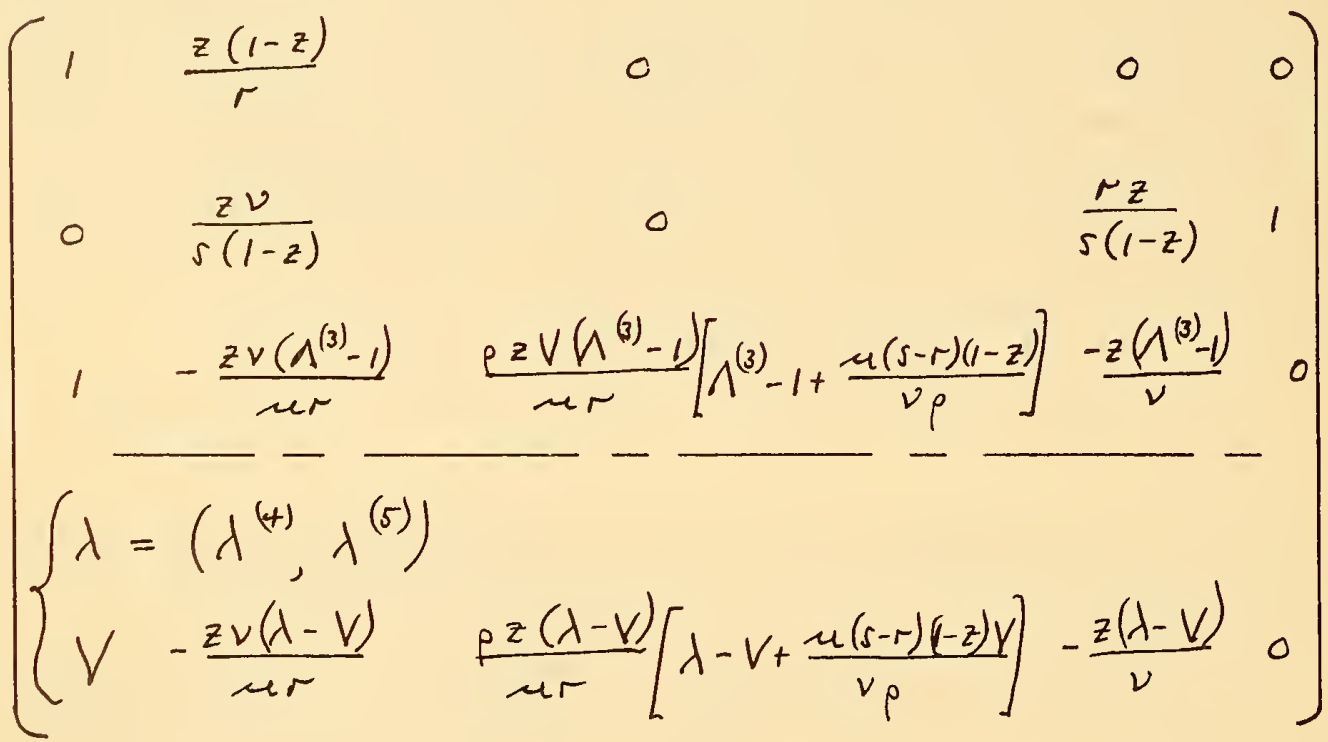

corresponding to eigenvalues:

$$
\lambda^{(i)}=\left(V, V, \Lambda^{(3)} V, \lambda^{(4)}, \lambda^{(5)}\right)
$$

20 
The first approach to the finite difference numerical integration was to use (58) and the computed inverse $Z^{i(j)}$ in the finite difference form of (56). Difficulties were encountered due to widely disparate orders of magnitude and "ill-conditioning" in (58). (The computed product generated diagonal elements near unity, but with some off-diagonal elements of the order $10^{3}$.) Rather than go to double precision, a satisfactory alternative was derived based on a fact earlier mentioned so that we may put

$$
\operatorname{sgn}\left(\lambda^{(j)}\right)=\operatorname{sgn}(V), \quad(j=1, \ldots, t)
$$

We may then rewrite (56) in the following form:

$$
\begin{aligned}
& u^{i},+b^{i j}\left\{\begin{array}{ll}
\left(u_{, x}^{j}\right)_{L} & (\alpha=1) \\
\left(u_{, x}^{\gamma}\right)_{R} & (\alpha=-1)
\end{array}\right\} \\
& +\alpha \beta\left(z^{i(5)} \lambda^{(5)}\right) \cdot\left(z^{(5) m}\left[\left(u_{, x}^{m}\right)_{R}-\left(u_{x}^{m}\right)_{L}\right]\right)=D^{i},
\end{aligned}
$$

where

$$
\begin{aligned}
& \alpha= \begin{cases}1 & (V \geqslant 0) \\
-1 & (V<0)\end{cases} \\
& \beta= \begin{cases}1 & \left(\lambda^{(5)} V<0\right) \\
0 & \left(\lambda^{(5)} V \geqslant 0\right)\end{cases}
\end{aligned}
$$

and $\left(z^{(5) m}, Z^{i(5)}\right)$ are left-and-right eigenvectors corresponding to $\lambda^{(5)}$. Specifically, we take 


$$
\left[\begin{array}{l}
z^{51}=V \\
z^{52}=\frac{1}{C^{34} C^{41}}\left[C^{34} c^{42}-C^{32}\left(C^{44}-\lambda^{(5)}\right)\right] \\
z^{53}=-\left(C^{44}-\lambda^{(5)}\right)\left(\lambda^{(5)}-V\right) \\
z^{54}=\frac{\lambda^{(5)}-V}{C^{41}} \\
z^{55}=0
\end{array}\right.
$$

and

$$
\left[\begin{array}{rl}
Z^{25} & =\frac{c^{23}}{c^{13}} Z^{15} \\
Z^{35} & =\frac{\lambda^{(5)}-V}{c^{12}} Z^{15} \\
Z^{45} & =\frac{\left(\lambda^{(5)}-V\right)^{2}-c^{32} c^{23}}{c^{13} C^{34}} \\
Z^{55} & =\frac{Z^{15}}{c^{13}\left(\lambda^{(5)}-V\right)}\left\{c^{13} c^{51}+c^{23} c^{52}\right. \\
& \left.+c^{53}\left(\lambda^{(5)}-V\right)+c^{5+}\left(\frac{\left(\lambda^{(5)}-V\right)^{2}-c^{23} c^{32}}{c^{34}}\right)\right\}
\end{array}\right.
$$

22 
For the "subsonic case", $\beta=1, \lambda^{(5)}$ has multiplicity 1 , so $z^{(5) j}$ and $Z^{i(s)}$ are orthogonal. Orthonormality gives the unknown scale $Z^{(s)}$ :

$$
z^{\prime(5)}=1 / \sum_{j=1}^{5} z^{(5) j}\left(\frac{z^{j(5)}}{z^{(5)}}\right)
$$

\section{Auxiliary Routines}

Primary emphasis so far has been on constructing a stable, controllable and flexible scheme "FIO4" for handling two-phase flows based on the hydrodynamic model (IV2T). Auxiliary factors, such as boundary conditions, initial conditions and sources terms, have not been intensively studied or varied except in so far as they have directly interacted with our primary purpose. To the extent that they have not interacted, simple representative types were taken for the auxiliary factors, and elaborated only to a degree that seemed necessary.

As far as was possible, the auxiliary factors have been isolated in subroutines to improve organization and facilitate changes.

In addition to the basic program (FIO4) using the CIR method with equations (60-64), three subroutines are used: BNDRY, INVC and KAPA.

Subroutine BNDRY is used to set values of the dependent variables on the end points, so that only interior points are treated by FIO4. In cases $I$ and II, BNDRY has been based on the assumption of an infinite reservoir at the left end, with source free, adiabatic flow from the right end at atmospheric pressure. Some studies were made with a finite tank BNDRY to determine tank pressure - surge pressure coupling, but this effect was determined not to be basic to the second surge formation.

Subroutine INVC computes and sets the initial values of the dependent variable (including $A(x)$ determined from a functional form) at both interior and end points.

The subroutine KAPA computes for interior points the source strength $e$ and $c$ and the source coefficients $D_{i j}$ of (38), including the friction terms $\left(\tau_{g}, \tau_{l}\right)$, the heat transfer rates $\left(q_{0}, q_{g}, q_{k}\right)$, the wetted perimeter per unit area $\left(k_{0}, k_{g}, k_{l}\right)$ and the non-linear 
pseudo-viscous stresses and work terms $\left(\mathscr{S}_{3}, \mathcal{S}_{+}, \mathcal{S}_{5}\right)$ via $(40-42)$.

The $\left(k_{0}, k_{g}, k_{l}\right)$ describe the character of the flow, $k_{0}$ indicating the degree of dispersion of the media. They are prescribed functions of the flow variables and geometry. In cases (I and II), we have taken

$$
k_{l}=(1-z) \hat{P}
$$

where $\widehat{P}$ is the pipe perimeter,

$$
k_{g}=z \hat{P}
$$

and

$$
K_{0}=2 K_{l}
$$

This gives volume-proportionate wall wetting in gas and liquid streams. Numerical experiments with annular flow, $k_{\ell}=\hat{P}, k_{g}=0, k_{0}=(1-z) \hat{P}$, showed sharply curtailed $\Delta t$ as $z \rightarrow 0$ because of large heat transfer into small quantities of liquid. Flow experiments indicate annular flow is not the cormon mode in cooldown, but that $k_{0} / K_{l}$ should be much larger.

The $\left(\tau_{g}, \tau_{l}\right)$ are taken as normal friction stresses:

$$
\begin{aligned}
& \tau_{g}=r v / v / f_{g} / 8 \\
& \tau_{l}=s v / v / f_{l} / 8
\end{aligned}
$$

where $f_{g}, f_{l}=.032$ for Reynolds number $\gamma_{l_{g}}, R_{l}$ greater than 2000 , and $f_{g}, f_{l}=\frac{64}{\left(R_{g}, R_{e}\right)}$ for Reynolds number below 2000. Here 


$$
\begin{aligned}
& R_{e_{g}}=\frac{r}{\sqrt{B(x)}} \frac{|V|}{\mu_{g}} \\
& \gamma_{e_{l}}=\frac{s}{\sqrt{B(x)}} \frac{|V|}{u_{l}}
\end{aligned}
$$

For the equation of state in the gas, we take

$$
p=A_{1}, T \quad\left(A_{1}=0.3(10)^{7}\right)
$$

with gas and liquid energies,

$$
\begin{aligned}
& E=C_{v g}\left(T_{g}-T_{c}\right) \\
& F=C_{v l}\left(T_{l}-T_{c}\right)
\end{aligned}
$$

and

$$
C_{v g}=0.81(10)^{7}
$$

We take the following linear approximations to the pressures and latent heat from data for $N_{2}$ on the vapor pressure curve:

$$
p_{v a} \equiv p_{v 1}+p_{v a} T=-0.138(10)^{8}+0.216(10)^{6} T
$$

and

$$
\lambda \equiv l_{1}+l_{2} T=2.98(10)^{9}-1.30(10)^{7} T .
$$

Since $\lambda=(E-F)_{T_{s_{0} t}}$, we find $\quad C_{v e}=0.211(10)^{8}$ and $T_{c}=230^{\circ}$.

25 
We take the heat transfer rates per $\mathrm{cm}^{2}$ per degree Kelvin from wall to gas $q_{q}^{*}$, from wall to liquid $q_{e}^{*}$, and from liquid to gas $q_{0}^{*}$ to be a simple form of the Dittus-Boelter correlation:

[4]

$$
\left\{\begin{array}{l}
q_{g}^{*}=q_{g}^{*} h_{g} \sqrt{B(x)} R_{g}^{0.8} \\
q_{l}^{*}=q_{l}^{*} h_{l} \sqrt{B(x)} R_{l}^{0.8}
\end{array}\right.
$$

and put

$$
q_{0}^{*}=q_{0}^{* *} h_{g} \sqrt{B(x)} R_{g}^{0.5}
$$

$$
\begin{aligned}
& h_{g}=0.1(10)^{*} \\
& h_{l}=0.13(10)^{5} \\
& q_{0}^{*}=q_{g}^{*}=q_{l}^{*}=0.023
\end{aligned}
$$

Then for heat sources we have

$$
\left\{\begin{array}{l}
k_{g} q_{g}=k_{g} q_{g}^{*}\left(T_{w}-T_{g}\right) \\
k_{l} q_{l}=k_{l} q_{l}^{*}\left(T_{w}-T_{l}\right) \\
k_{0} q_{0}=k_{0} q_{0}^{*}\left(T_{l}-T_{g}\right)
\end{array}\right.
$$

Since we are using only bulk heat transfers and mean temperatures, no precise estimates of evaporation or condensation at the interface can be made. Such an estimate would depend on temperature gradients at the interface, and the relative values of interface temperature and interface saturation temperature. We distinguish three sources of evaporation and condensation: $\left(e_{1}, c_{1}\right)$ at interface, $\left(e_{2}, c_{2}\right)$ at wall, $\left(e_{3}, c_{3}\right)$ spontaneous for supersaturated cases. Finally,

$$
(e, c)=\left(e_{1}, c_{1}\right)+\left(e_{2}, c_{2}\right)+\left(e_{3}, r_{3}\right)
$$

26 
Then we have used the following forms:

$$
\begin{aligned}
e_{1}= \begin{cases}0 & \left(z \geqslant z_{\text {max }}\right) \\
0 & \left(T_{g} \leqslant T_{\text {sat }}(p)\right) \\
\sigma_{1} \frac{k_{0} q_{0}^{*}\left(T_{g}-T_{\text {sat }}\right)}{q_{x}} A & \left(T_{g}>T_{\text {sat }}(p)\right)\end{cases} \\
c_{1}= \begin{cases}0 & \left(z \leqslant z_{\text {min }}\right) \\
0 & \left(T_{\text {sat }} \leqslant T_{l}\right) \\
\sigma_{+} \frac{k_{0} q_{0}^{*}\left(T_{\text {sat }}-T_{c}\right)}{q_{x} A} & \left(T_{s a t}>T_{l}\right)\end{cases}
\end{aligned}
$$

where

$$
\begin{aligned}
& q_{x}=\max \cdot\left\{E-F, 0.25(10)^{8}\right\} \\
& e_{2}= \begin{cases}0 & \left(z \geqslant z_{\text {max }}\right) \\
0 & \left(T_{\omega} \leqslant T_{\text {sat }}\right) \\
\sigma_{2} \frac{k_{l} q_{l}^{*}\left(T_{\omega}-T_{\text {rat }}\right)}{q_{x}} A & \left(T_{\omega}>T_{\text {sat }}\right)\end{cases}
\end{aligned}
$$

and

27 


$$
c_{3}= \begin{cases}0 & \left(z \leqslant z_{\text {min }}\right) \\ 0 & \left(T_{s a t} \leqslant T_{w}\right) \\ \sigma_{s} \frac{k_{g} q_{g}^{*}\left(T_{s a t}-T_{w}\right)_{A}}{q_{x}} & \left(T_{s a t}>T_{w}\right)\end{cases}
$$

The $\sigma_{j}$ are "efficiencies" of conversion of transferred heat to evaporation and condensation. Also,

$$
e_{3}= \begin{cases}0 & \left(z \geqslant z_{\text {max }}\right) \\ 0 & \left(T_{l} \leqslant T_{\text {sat }}\right) \\ \sigma_{3}(1-z)\left(T_{l}-T_{\text {sat }}\right) A & \left(T_{l}>T_{\text {sat }}\right)\end{cases}
$$

and

$$
c_{3}= \begin{cases}0 & \left(z \leqslant z_{\text {Mad }}\right) \\ 0 & \left(T_{g} \geqslant T_{\text {sat }}\right) \\ \sigma_{6} z\left(T_{\text {sat }}-T_{g}\right) A & \left(T_{g}<T_{\text {sat }}\right)\end{cases}
$$

We have used $\sigma_{j}=0.5,(j=1,2,4,5)$, and $\sigma_{j}=0.001$ for $(j=3,6)$. This latter was chosen simply because it gave quantities for $\left(e_{3}, e_{3}\right)$ of the same order as had been obtained in typical cases for $\left(e_{2}, c_{2}\right)$.

In the calculation of $(4 I, 42)$ the calculation of $\frac{\partial V}{\partial x}$ was modified 
to

$$
\left(\frac{\partial V}{\partial x}\right)=\frac{\partial V}{\partial x}-\frac{Z V}{\left(\frac{\rho z V^{2}}{\gamma p}-1\right)}
$$

the minuend being chosen to remove the part of the derivative due to variable geometry, as computed from the steady adiabatic flow equations:

$$
\begin{aligned}
& (A(1-z) V)_{x}=0 \\
& (A z+V)_{, x}=0 \\
& \left(A z r^{2}+A(1-z)_{s} V^{2}+A p\right)_{, x}=p A, x \\
& \left(p r^{-\gamma}\right)_{x}=0
\end{aligned}
$$

In the equation (7) for wall heat transfer, the heat transfer rate was taken to be

$$
h_{\omega x}=\frac{\hat{P}_{A}}{\pi \delta_{\rho \omega} C_{p \omega}}=0.22(10)^{-6}
$$

where $\hat{P}_{A}$ is the perimeter of the uniform pipe, $\delta$ its thickness, $\rho_{w}$ its mass density, and $C_{\text {pw }}$ its heat capacity.

\section{Controls and Blocks}

This finite difference scheme, called FLO4, is finally then in the form

$$
u^{i}(x, t+\Delta t)=u^{i}(x, t)+\Delta t\left(D^{i}(x, t)-E^{i}(x, t)\right)
$$

At least three complete passes through the net of points in $\mathrm{x}$ are involved in FLO4 for a single time step. On the first pass, boundary values are set, and $(\Delta t)_{\max }$, satisfying $(31)$, is obtained. We also require $\Delta t$ to be less than twice the value of $\Delta t$ used on the previous time line. $D^{i}$ and $E^{i}$ are also computed. On the second pass, $\left(u^{i}\right)^{+} \equiv u^{i}(x, t+\Delta t)$ is found at each 
$x$, and tested to be certain that the following limits are satisfied:

$$
\left\{\begin{array}{l}
\left|z^{+}-z\right| \leqslant(U L Z) \cdot|z| \\
\left|r^{+}-r\right| \leqslant(U\llcorner R) \cdot|r| \\
\left|V^{+}-V\right| \leqslant \max ((U L V),(U L U) \cdot|V|) \\
\left|T_{g}^{+}-T_{g}\right| \leqslant(U L G) \cdot\left|T_{g}\right| \\
\left|T_{l}^{+}-T_{l}\right| \leqslant(U L L) \cdot\left|T_{l}\right|
\end{array}\right.
$$

Tested for positivity is the argument of the logarithm in (92). If any of these tests fail, we reset $\Delta t=\frac{1}{2} \Delta t$, and repeat the second pass. In the event that the computed value $z^{+}$falls outside the range $z_{\text {MIN }} \leqslant z \leqslant z_{\text {MAX }}$, we set $z^{+}$to the nearer of these two limits, and use equation (32) in reverse to solve for the $c$ or $e$ (positivevalued) required. This latter value is used in the remaining equations. In case $I$ and II, we have used

$$
\left\{\begin{array}{l}
U L Z=U L R=U L U=U L G=U L L=0.1 \\
U L V=0.1(10)^{4} \\
z_{M 1 N}=0.05, \quad z_{M A X}=e^{-10}
\end{array}\right.
$$

In the third pass, the new values of the dependent variable $\left(z^{+}(t, x)\right.$, etc.) are stored as old values $(z(t, x)$, etc.) in preparation for computing a new line. BNDRY is used here.

In view of the fact that 1 - $z$ must be permitted to become very small, we have used $1-z \equiv e^{-\sigma}$ in all algebraic expressions, and replaced

$$
\Delta z=F^{*} \cdot \Delta t
$$

by its exact equivalent

$$
\Delta \sigma=-\ln \left(1-e^{\sigma} \Delta t \cdot F^{*}\right)
$$

and similarly for $\mathrm{x}$ - derivatives. 


\section{Computation Summary}

Computations were carried out on an IBM 7090 with an 8-digit floating point mantissa. The finite difference scheme based on (60-64) appears to be stable and to give solutions of the proper character during the growth and early stages of decay of the first surge, a real time of about 2 seconds. In Case I, a peak pressure of 17 atmospheres was calculated. For these computations, $\Delta \mathrm{x}=290 \mathrm{~cm}$ was taken (20 stations in the pipe length), yielding a computing rate of 0.64 flow seconds per minute of computing. Since flow times of the order of 10 seconds or more are desired ( 16 minutes computing), it does not seem reasonable to reduce $\Delta x$ significantly, especially since cutting $\Delta x$ by two quadruples the computing time for a given real time interval.

After the first surge, liquid was flowing back into the tank, and gas was flowing rapidly from the down stream end of the pipe. The pressure fell rapidly until it began to near the tank pressure. At this level, the pressure no longer fell, but was sustained partly by boiling of a non-diminishing liquid residue in the pipe and by non-diminishing gas energy and gas density. A quasi-steady state thus resulted, and no second surge evidenced itself.

The reason that energy, pressure, and liquid phase are maintained in spite of the rapid transport of these quantities out of the pipe at each end can be seen by writing the equations (60) in the form

$$
\begin{gathered}
u_{, t}^{i}+b^{i j}\left\{\begin{array}{ll}
\left(u_{, j}^{j}\right)_{L} & (\alpha=1) \\
\left(u_{, j}^{j}\right)_{R} & (\alpha=-1)
\end{array}\right\}-D^{i} \\
=-\alpha \beta z^{i(5)} \lambda^{(5)} z^{(5) j}\left(\frac{\partial^{2} y^{j}}{\partial x^{2}}\right) \Delta x \cdot F(i) \\
+C\left((\Delta x)^{2}\right)
\end{gathered}
$$


and

$$
F^{i}=1 . \quad(\text { Case I) }(94 a)
$$

where we have approximated the difference in right- and left-hand derivatives by a second derivative within the accuracy of the finite difference approach. The equations (93) are diffusion equations, $-\alpha \lambda^{(5)} z^{i(5)} z^{(5) i} \geqslant 0$, the diffusion terms in the right-hand side tending to smooth out irregularities in the functions and their derivatives. Under the smoothness conditions of the CIR proof, these terms vanish as $\Delta x \rightarrow 0$, but remain large enough to stabilize the numerical method. In the computation described above, however, these terms dominate the convective terms on the left-hand side when the pressure has fallen to nearly tank pressure. This diffusion also probably accounts in part for the earlier surge peak times encountered. This numerical diffusion is, of course, a physical interpretation of the effect of truncation involved in the finite difference equations.

A few attempts have been made to circumvent these difficulties, and allow for the formation of multiple surges without reducing the stability of the system. The only moderately successful one was to introduce multipliers which tended to reduce the diffusion when it was already small:

$$
F^{i}=\frac{\left|\left(u_{, x}^{i}\right)_{R}-\left(u_{, x}^{i}\right)_{L}\right|}{\left|\left(u, u_{, x}\right)_{R}\right|+\left|\left(u_{, x}^{i}\right)_{L}\right|}
$$

Then $0 \leqslant F^{i} \leqslant 1$, and $F^{i}=1$ if the right- and left-hand derivatives have opposite signs. Using this form (Case II) a small second surge was observed, while the peak pressures in the first surge reached only 11 atmospheres.

Because so severe a flow change appears to result from adjustment of purely computing parameters, it might on first sight seem to invalidate 
both results, but the diffusion is unquestionably overvalued in each case. Any scheme which retains stability while reducing the diffusion should give improved results. It might well be worth testing other ideas in this regard. Two alternatives are ( 1 ) greatly reducing $\Delta x$, giving a practical computing method only for problems in a very small geometry or a very short time, or (2) attempting to program a Lagrangian scheme, in which the diffusion required for stability might be much less, and better liquid control might be maintained. It does seem that because of the high mass, energy and momentum density of the liquid, a good representation of liquid movement is essential to a meaningful computing scheme.

It should be noted that in (93), $F^{l}$, the factor in the liquid continuity $(z)$ equation, can be set to zero, or even made negative without interfering with the stability of the scheme, so long as $0<z<1$ is retained. In some tests here, further improvements have been made in avoiding liquid diffusion, but results are not conclusive. Making $\mathrm{F}^{\mathrm{I}}$ dependent on flow-direction and $\frac{\partial^{2} z}{\partial x^{2}}$, has also been tried inconclusively. Some realism might be gained here, in reducing the requirement of continuity in the liquid, and emphasizing transport. (Indeed, for small $\mathrm{z}$ with droplets, $\mathrm{z}$ - waves cannot propagate.)

Cases I and II were computed with the initial values: (CGS system)

$\begin{array}{llc} & \frac{\Delta x \leqslant x \leqslant 5 \Delta x}{V} & 5 \Delta x<x \leqslant 20 \Delta x \\ B(x) & \frac{0.278^{0}}{16(\Delta x)^{2}}(x)(9 \Delta x-x) & 0.278 \\ p & 0.5065(10)^{7} & 0.1013(10)^{7} \\ r & 0.2412(10)^{-1} & 0.1125(10)^{-2} \\ z & 0.05 & 0.99995 \\ T_{g} & 70^{\circ} \mathrm{K} . & 300^{\circ} \mathrm{K} . \\ T_{e} & 70^{\circ} \mathrm{K} . & 65^{\circ} \mathrm{K} . \\ T_{w} & 70^{\circ} \mathrm{K} . & 300^{\circ} \mathrm{K} .\end{array}$


with the single addition $T_{w}(6 \Delta x, 0)=70^{\circ} \mathrm{K}$. to admit heat leakage. Another variation was made on FIO4 (using $\mathrm{F}^{i}=1$ ) to test a model of valve opening time $T$ and to attempt to match computationally the strong dependence of peak pressure on valve-opening time observed experimentally. While not thoroughly understood, this dependence may be due to liquid jet formation at the valve. If this were so, the effect would be due then to spatial variation which cannot be expected to come out of a one-dimensional analysis. We used

$$
\begin{cases}B(x, t)=B(x) \frac{t}{T} & (0 \leqslant t \leqslant T) \\ B(x, t)=B(x) & (t \geqslant T)\end{cases}
$$

An increase of peak pressure did occur as opening time increased, but this trend did not reverse itself for large opening times as is observed experimentally. Again the liquid diffusion dominated transport when the valve opened slowly; the liquid that was thus introduced downstream of the valve boiled, and the valve restrictions prevented the required back flow which would have prevented the sky-rocketing pressure at 10 seconds. Furthermore, the valve was represented by a transient area variation which was not small and stationary as was assumed in the derivation of the equations.

Again, finer difference nets would have permitted better valve representation without the large area variations introduced here: Valve Opening Time (Sec)

$\begin{array}{cc}\text { Peak Pressure (Atmosphere) } & \text { Time } \\ 13.8 & 0.30 \\ 15.9 & 0.49 \\ 19.3 & 0.59 \\ 39.0 & 0.43 \\ ? & \end{array}$

(See figures 3, 4, and 5.)

In Figures 1 through 5 , the pressure $p$, volumetric quality $z$, and velocity $V$ are plotted at each of the twenty pipe stations for times given by the baselines (arrows on time scale). Above each baseline, $k$ is measured in atmospheres (absolute)/o.I inch, $\mathrm{z}$ in units/inch, V in 1000 
$\mathrm{cm} / \mathrm{sec} / 0.1$ inch.

Figure 6 shows the pressure in atmospheres for Case $I$ (lower) and Case II (upper) as a function of time for station $7(x=7 \Delta x)$, the location of the pressure peak in each case. The circles are at the points in time for which results were printed, less frequent in Case I.

Figure 7 is an experimental curve given in a private communication. Note the spread in experimental data.

Clearly, the worst defects of the FIO4 model are in time of first peak and peak decay rates. While perhaps these could be improved by modifying the geometry $B(x)$ and improving the empirical heat and momentum transfer formulae, it is certain that the numerical diffusion is the major culprit. With the present costs of high speed computing, it is not practical to use $\Delta x$ small enough to evaluate the model's potential effectiveness in predicting the Cooldown process.

ACKNOWLEDGEMENT The author wishes to express his gratitude to Messrs. R. V. Smith and W. G. Steward for their cooperation and advice, and to $\mathrm{Mr}$. J. H. Wilson for many useful discussions and invaluable aid in programming. 


\section{Glossary}

Physical quantities are measured in CGS units. Subscripts $g, \boldsymbol{l}, \mathrm{o}$ refer respectively to gas (or wall-to-gas), liquid (or wall-to-liquid) and interface (or liquid-to-gas), and are replaced below by the generic subscript $\varphi$. Units are shown in square brackets. $a_{i j}$ : coefficient matrix of time derivatives for quasilinear hyperbolic system.

$A_{1}$ : gas constant in state equation for perfect gas. (eq.71) $A(x)$ : cross-sectional area of pipe $(A(x, t)$ used in study of effect of valve-opening time). $\left[\mathrm{cm}^{2}\right]$

$b^{i j}$ : coefficient matrix of $x$-derivatives for quasilinear hyperbolic system. $B(x): I / A(x) \cdot\left[\mathrm{cm}^{-2}\right]$

$c, c_{i}$ : mass rates of condensation per unit length of pipe. (eq.78) $\left[\frac{\mathrm{gm}}{\mathrm{cm} \cdot \mathrm{sec}}\right]$ $\mathrm{C}_{\mathrm{v}}$ : specific heat at constant volume. $\left[\frac{\mathrm{crgs}}{\mathrm{gm} .{ }^{\circ} \mathrm{K}}\right]$

$\mathrm{C}_{\mathrm{pw}}$ : specific heat of. pipe wall. $\left[\frac{\mathrm{ergs}}{\mathrm{gm} .{ }^{\circ} \mathrm{K}}\right]$

$D_{i}, D_{i j}$ : source terms for (IV2T) ${ }_{C}$, with partition. (eqs.37,38)

$e, e_{i}$ : mass rates of evaporation per unit length of pipe. (eq.78) $\left(\frac{\mathrm{gm} .}{\mathrm{cm} \text { sec. }}\right)$ $\mathrm{E}$ : specific energy of gas. (eq.72) $\left[\frac{\mathrm{ergs}}{\mathrm{gm} .}\right]$

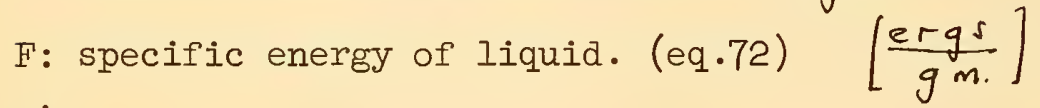
$\mathrm{F}^{\mathrm{i}}$ : a non-linear diffusion coefficient. (eqs.94a,94b) [1]

$F^{*}$ : typical time derivative. (eqs.91,92 only)

$f_{\varphi}$ : friction factor. [1]

$g(x)$ : component of gravitational acceleration along pipe. $\left[\frac{\mathrm{cm} .}{\mathrm{sec}^{2}}\right]$ $\mathrm{h}_{\mathrm{wX}}$ : wall heat parameter. (eq.87) $\left[\frac{\mathrm{cm}^{3}{ }^{\circ} \mathrm{K}}{\mathrm{erg}}\right]$ $\mathrm{h}_{\varphi}:$ heat conductivity. $\left[\frac{\mathrm{ergs}}{\mathrm{cm} \cdot \mathrm{sec}^{\circ} \mathrm{K} .}\right]$ $\mathrm{k}_{\varphi}: \ell_{\varphi} \mathrm{B} \cdot(\mathrm{eq} \cdot 39) \quad\left[\mathrm{cm}^{-1}\right]$ 
$\ell_{\varphi}$ : perimeter of contact on pipe cross-section. (eqs.10-13) [cm] $p:$ pressure. $\left[\frac{d y n c r}{\mathrm{~cm}^{2}}\right]$

$p_{\mathrm{va}}(\mathrm{T})$ : vapor pressure. (eq.73) $\left[\frac{\text { dyncrs }}{\mathrm{cm}^{2}}\right]$

$P(r, E)$ : general equation of state for vapor. (eq.8)

$\hat{P}(x), \hat{P}_{A}$ : pipe perimeter, and its value on uniform section. [cm] $q_{x}:$ (eq.81) $\left[\frac{\text { ergs }}{g_{m},}\right]$

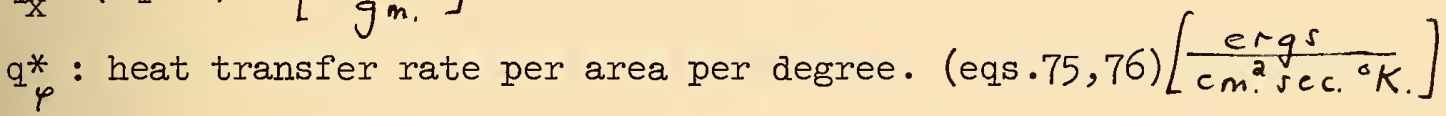

$\mathrm{q}_{\varphi}^{* *}$ : constants in Dittus-Boelter correlation. (eq.75,76) [1]

$q_{\varphi}$ : heat transfer rate per unit area. (eq.77) $\left[\frac{\mathrm{ergs}}{\mathrm{cm} \mathrm{g}^{2} \mathrm{sec}}\right]$

$q_{\varphi}:$ pseudo-viscous stress. (eqs. 41,42) $\left[\frac{\frac{d y n c r}{\mathrm{~cm}^{2}}}{\mathrm{c}^{2}}\right]$

$r$ gas density. $\left[\frac{\mathrm{gm}_{\mathrm{m}}}{\mathrm{cm}^{3}}\right]$

$\mathrm{R}_{\varphi}$ : Reynolds Number. (eq.70)

$\mathrm{s}$ : liquid density. $\left[\frac{\mathrm{gm} .}{\mathrm{cm}^{3}}\right]$

$\mathrm{S}_{1}$ : sources. (eqs.9-14)

$\mathcal{f}_{i}$ : sources. (eq.40)

$\mathrm{t}$ : time. [sec]

T: valve-opening time. (eq.95) [sec]

$\mathrm{T}_{\text {sat }}(p)$ : saturation temperature, inverse of $\beta_{\mathrm{va}}(\mathrm{T}) \cdot\left[{ }^{\circ} \mathrm{K}\right]$

$\mathrm{T}_{\varphi}$ : fluid temperature. [ $\left.{ }^{\circ} \mathrm{K}\right]$

$\mathrm{T}_{\mathrm{W}}$ : wall temperature. $\left[{ }^{\circ} \mathrm{K}\right]$

$\mathrm{T}_{\mathrm{c}}$ : base temperature for eqs. $72 .\left[{ }^{\circ} \mathrm{K}\right]$

$u^{i}$ : dependent variable vector: $(z, r, V, E, F)$

$\left(u^{i},{ }_{X}\right)_{R},\left(u^{i},{ }_{x}\right)_{L}:$ right- and left-hand $x$-derivatives.

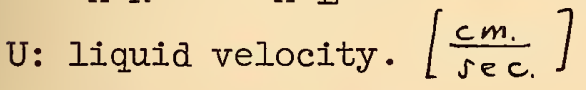

UIZ, UIR, ULU, ULV, ULG, ULL: limits. (eq.89)

$\mathrm{V}$ : gas velocity. [ $\left.\frac{\mathrm{cm} .}{\mathrm{sec}}\right]$ 
$\mathrm{x}$ : coordinate along pipe axis. $[\mathrm{cm}]$

$y$ : quality (mass of gas per mass of fluid mixture). [1]

z: fraction of pipe cross-section containing gas. [1]

$\mathrm{Z}$ : pipe expansion rate. (eq.25) $\left[\mathrm{cm}^{-1}\right]$

$\mathrm{z}_{\text {MAX }}, \mathrm{z}_{\text {MIIN }}$ : bounds on admissible $\mathrm{z}$. (eq. 90)

$\mathrm{z}^{i j}, \mathrm{z}^{i j}$ : matrices of left and right eigenvectors. (eqs.51,57,63, 64)

$\gamma$ : ratio of specific heats in gas phase. [1]

$\delta$ : pipe wall thickness. $[\mathrm{cm}]$

$\delta^{i j}$ : Kronecker delta.

$\Delta \mathrm{x}$ : fixed space increment. $[\mathrm{cm}]$

$\Delta t$ : variable time increment. (eq.31) [sec]

$\lambda^{i}$ : fluid signal speeds. $\left[\frac{\mathrm{cm} .}{\mathrm{sec}}\right]$

$\lambda^{*}$ : latent heat of vaporization. (eq.74) $\left[\frac{\mathrm{ergs}}{\mathrm{gm} .}\right]$

$\mu_{\varphi}$ : viscosity. $\left[\frac{g m .}{\mathrm{cm} . \mathrm{sec} .}\right]$

$\mu:\left(\right.$ eqs.16,46) $\left[\frac{\mathrm{ergs}}{\mathrm{gm} .}\right]$

$\nu:\left(\right.$ eqs.16,46) $\left[\frac{\mathrm{ergr}}{\mathrm{gm} .}\right]$

$\rho$ : mean density. (eq. 3I) $\left[\frac{\mathrm{gm} .}{\mathrm{cm}^{3}}\right]$

$\rho_{\mathrm{w}}$ : pipe wall density. $\left[\frac{\mathrm{gm} \cdot \mathrm{cm}^{3}}{\mathrm{c}^{3}}\right]$

$\sigma=-\ln (1-z)[1]$

$\sigma_{i}$ : coefficients in formulae (79-83). [1]

$\tau_{\varphi}$ : shear stresses. (eq.69) $\left[\frac{d y n e s}{c m .^{2}}\right]$

$\omega:($ eq.16) [1]

(2V2T): system of conservation equations (1-8) allowing each fluid a veloeity and temperature.

$(\text { IV2T })_{A}$ : system of conservation equations with one velocity obtained by replacing $(3,4)$ in $(2 \mathrm{~V} 2 \mathrm{~T})$ by $(23,24)$ 
$(I \mathrm{~V} 2 \mathrm{~T})_{\mathrm{B}}$ : system of modified conservation equations (26-30) with one velocity.

$(\text { IV2T })_{C}$ : system of quasilinear equations (32-36) with one velocity used in FIO4 numerical evaluations. 


\section{References}

1. R. Courant, E. Isaacson, M. Rees, "On the Solution of Non-Iinear Hyperbolic Differential Equations by Finite Differences," CPAM V, pp. 243-255 (1952).

2. R. D. Richtmyer, "Difference Methods for Initial Value Problems," Interscience, 1957.

3. G. Forsyth and W. Wasow, "Finite Difference Methods for Partial Differential Equations," Wiley, 1960.

4. F. W. Dittus, and L. N. K. Boelter, Univ. of Cal. Pubs., Eng. Vol. 2, p. 443, 1930. 
Appendix

The Finite Difference Method of Lax.

Lax's method assumes a system of hyperbolic partial differential equations in "Conservation Law" form:

$$
f_{, t}^{i}+g_{, x}^{i}+h^{i}=0
$$

where, for our cases, $\left(f^{i}, g^{i}, h^{i}\right)$ are algebraic functions of the flow variables $\left(z, r, V, T_{g}, T_{l}\right)$. The computation is advanced in time from a given line of values given at equally spaced $(2 \Delta x)$ values of $x$ on a staggered net by substituting in (A-I) the following expressions:

$$
f_{t}^{i}(x, t) \doteq \frac{f^{i}(x, t+\Delta t)-\frac{1}{2}\left[f^{i}(x+\Delta x, t)+f^{i}(x-\Delta x, t)\right]}{\Delta t}
$$

and

$$
g_{x}^{i}(x, t)=\frac{g^{i}(x+\Delta x, t)-g^{i}(x-\Delta x, t)}{2 \Delta x}
$$

After computing a line, the flow variables are computed from the $f^{i}(t+\Delta t, x)$. On theoretical grounds, we must have that $\Delta t / \Delta x$ be less than the maximum signal speed occurring in the flow at time $t$. The averaging resulting from the average used in (A-2) is necessary to the stability of the method, but causes too great a diffusion of flow quantities in the Cooldown problem to be useful. There does not appear to be a rigorous theory of convergence and stability for the lax method comparable to that given for the CIR method (39). 



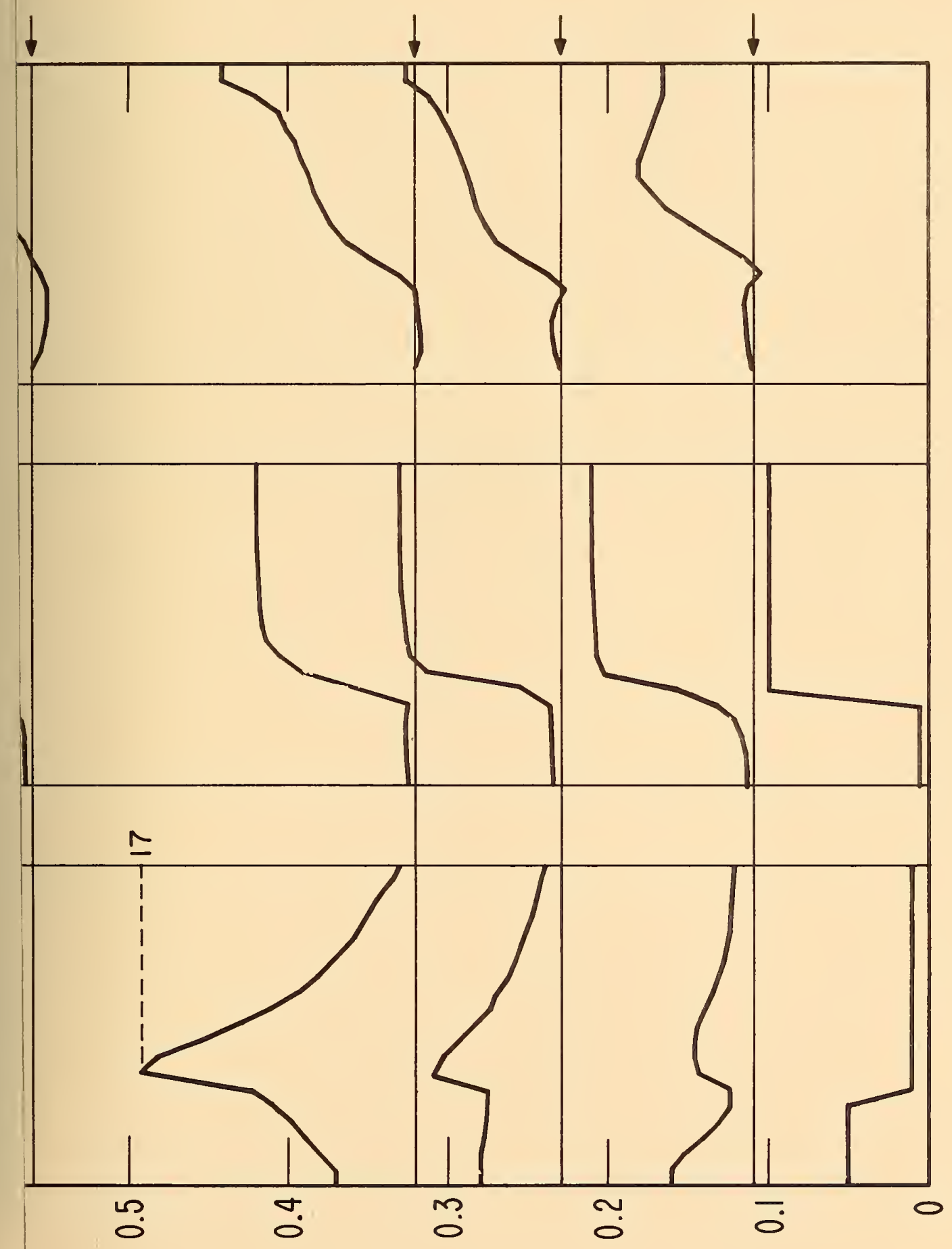

-
$\frac{1}{5}$
$\frac{5}{4}$ 



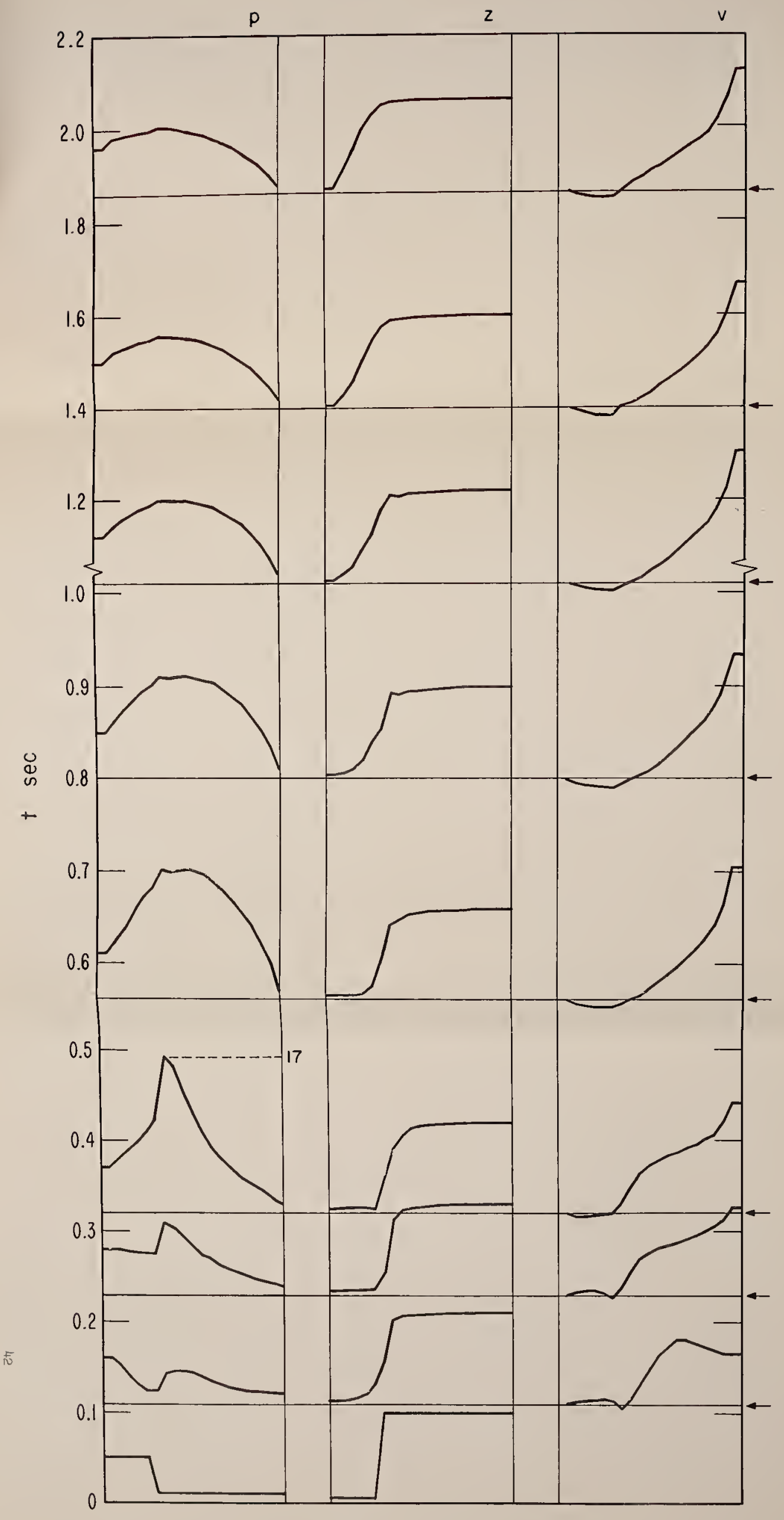

Figure I 


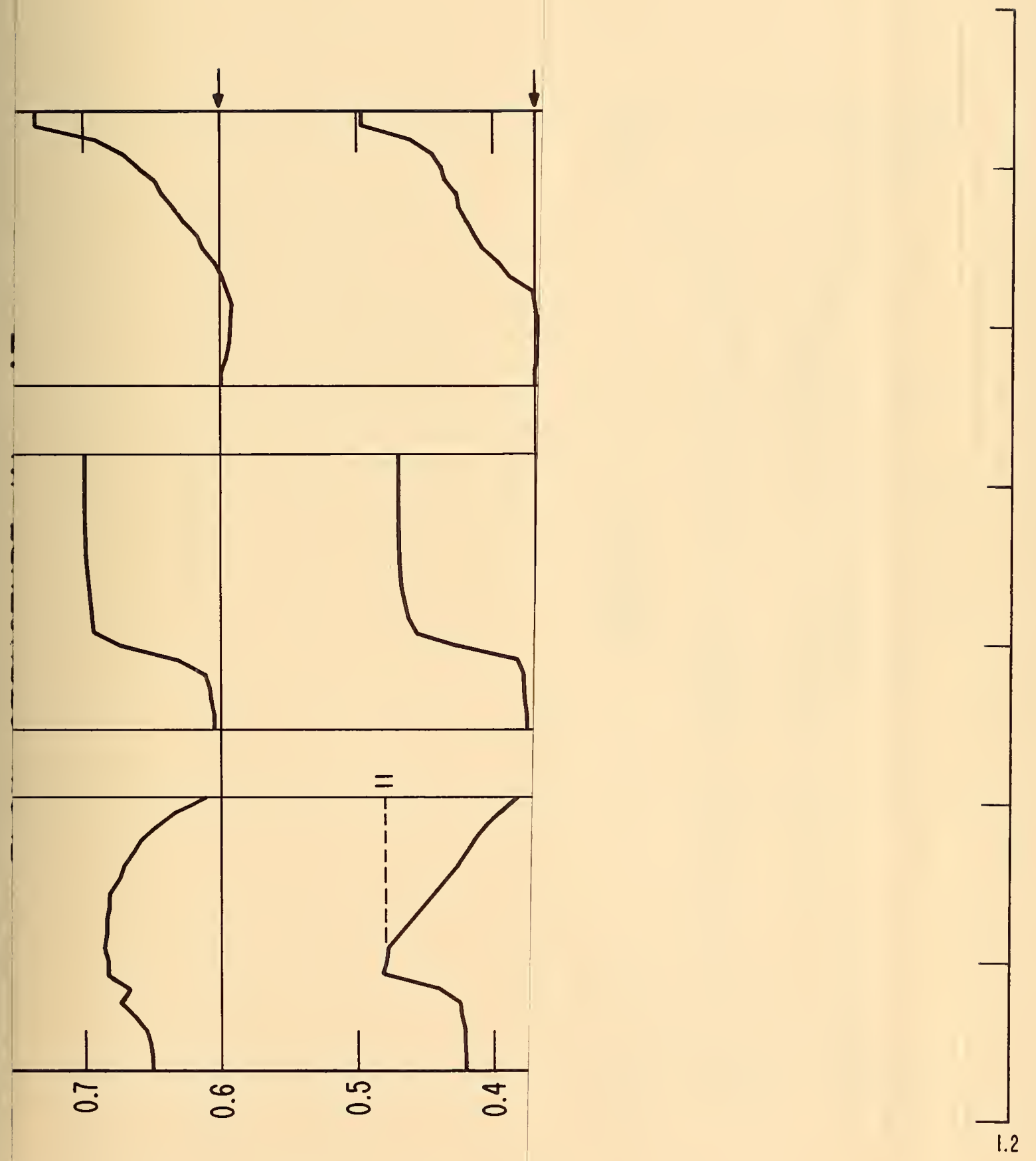

FLOW STRUCTURE IN $X$ AT

A SEQUENCE OF TIMES

CASE II: $F^{i} \neq 1$ (SEE Eq. 94b)

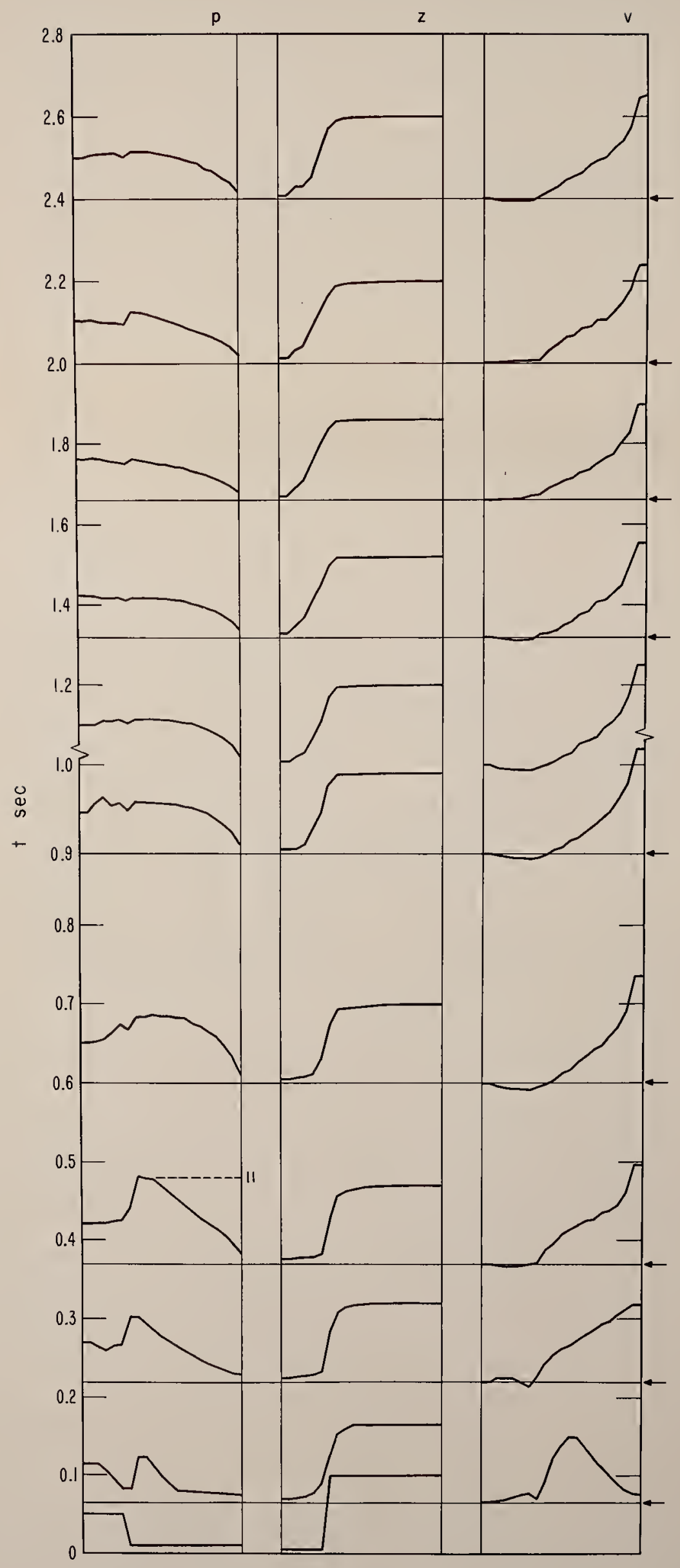

Figure 2 



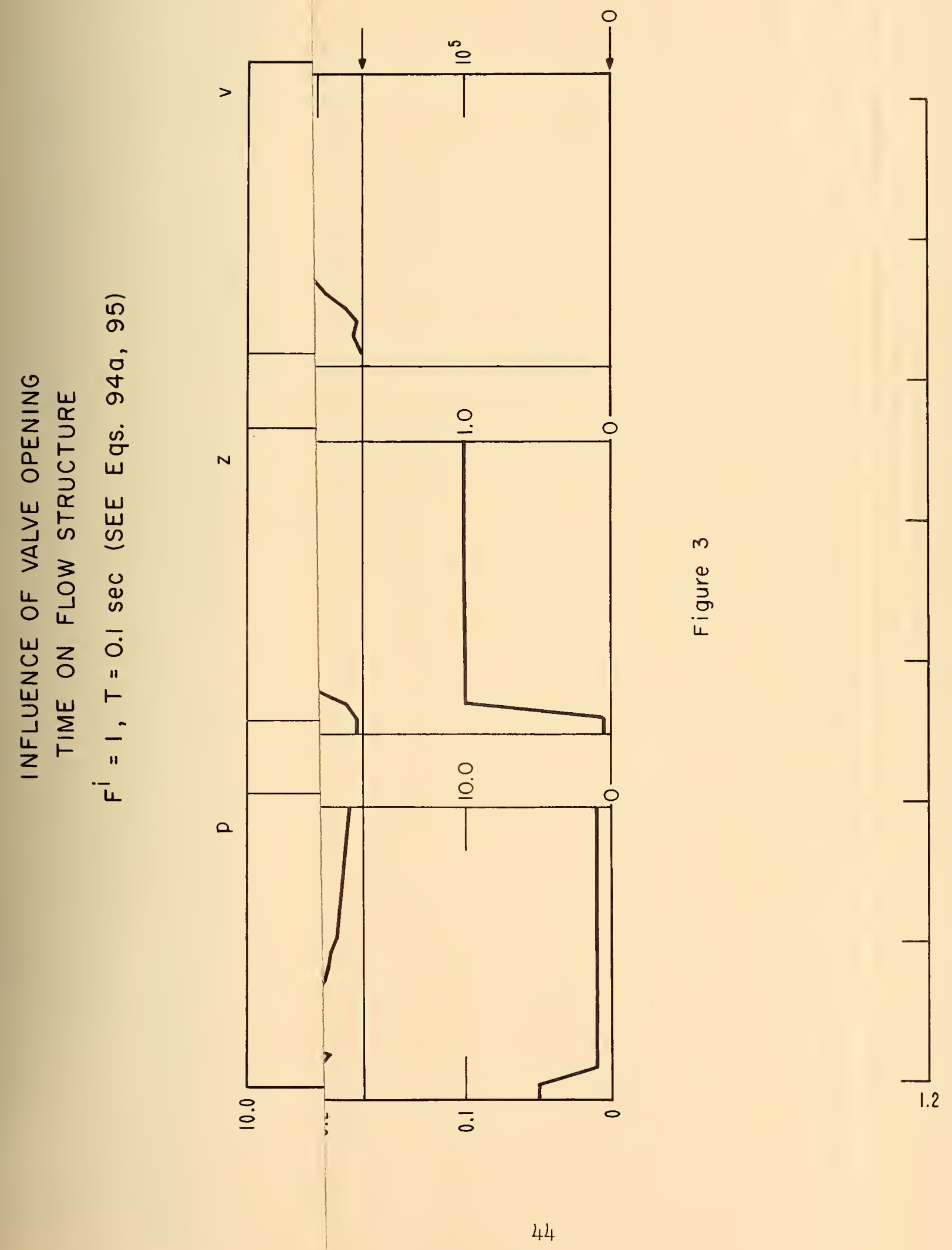



INFLUENCE OF VALVE OPENING

TIME ON FLOW STRUCTURE

$F^{i}=1, T=0.1$ sec (SEE Eqs. 94a, 95)

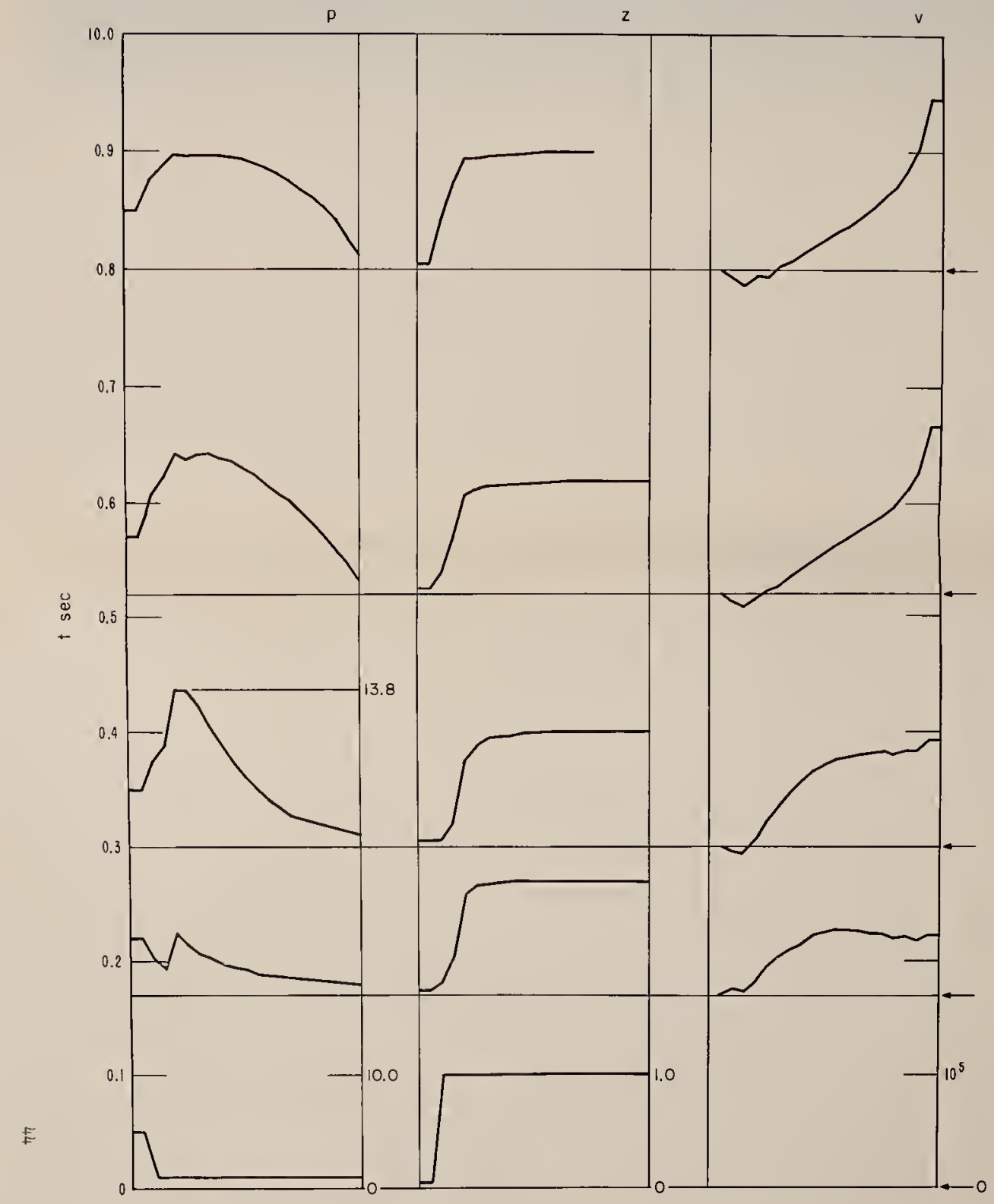

Figure 3 



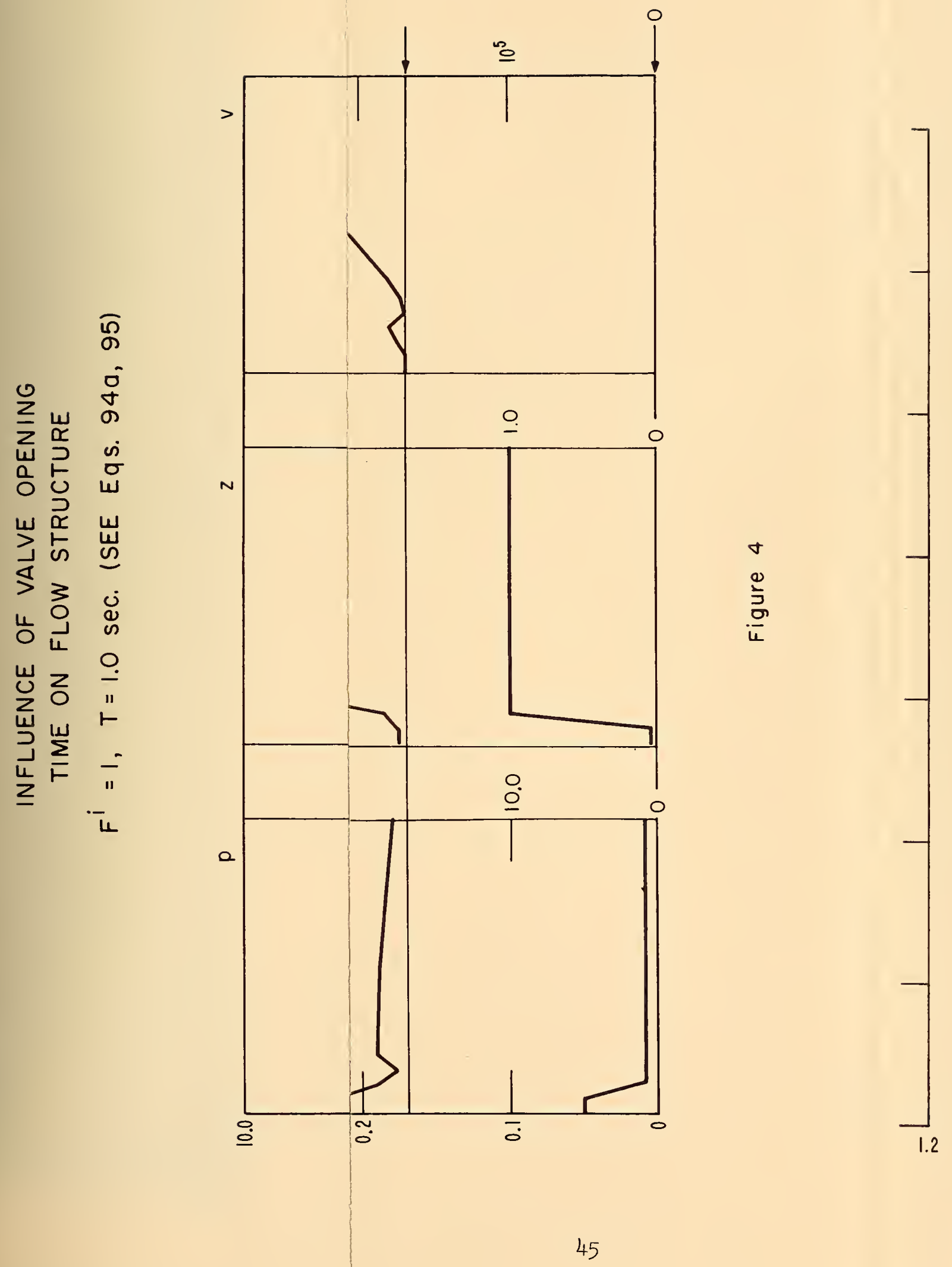



INFLUENCE OF VALVE OPENING

TIME ON FLOW STRUCTURE

$F^{i}=1, T=1.0 \mathrm{sec}$. (SEE Eqs. 940,95 )

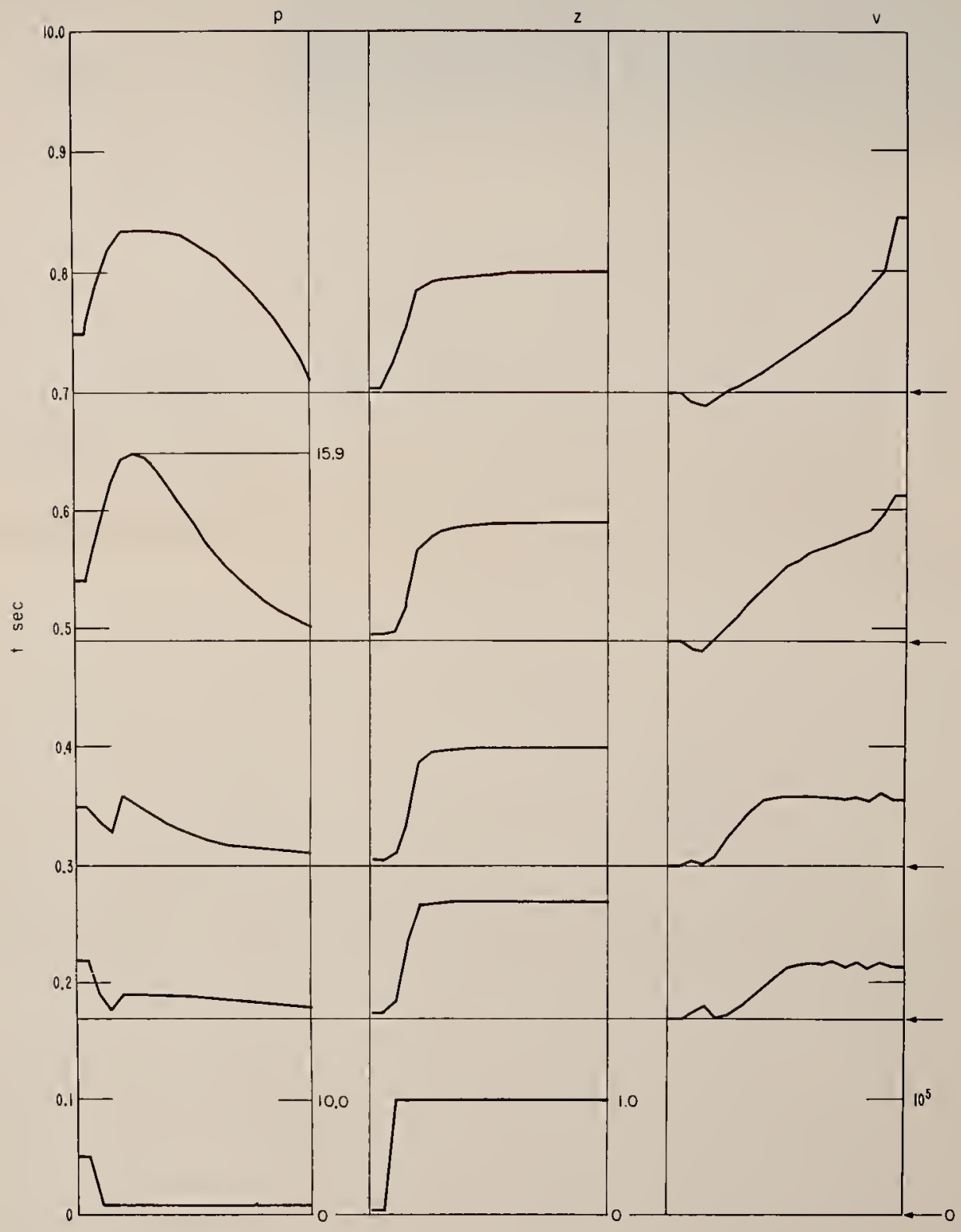





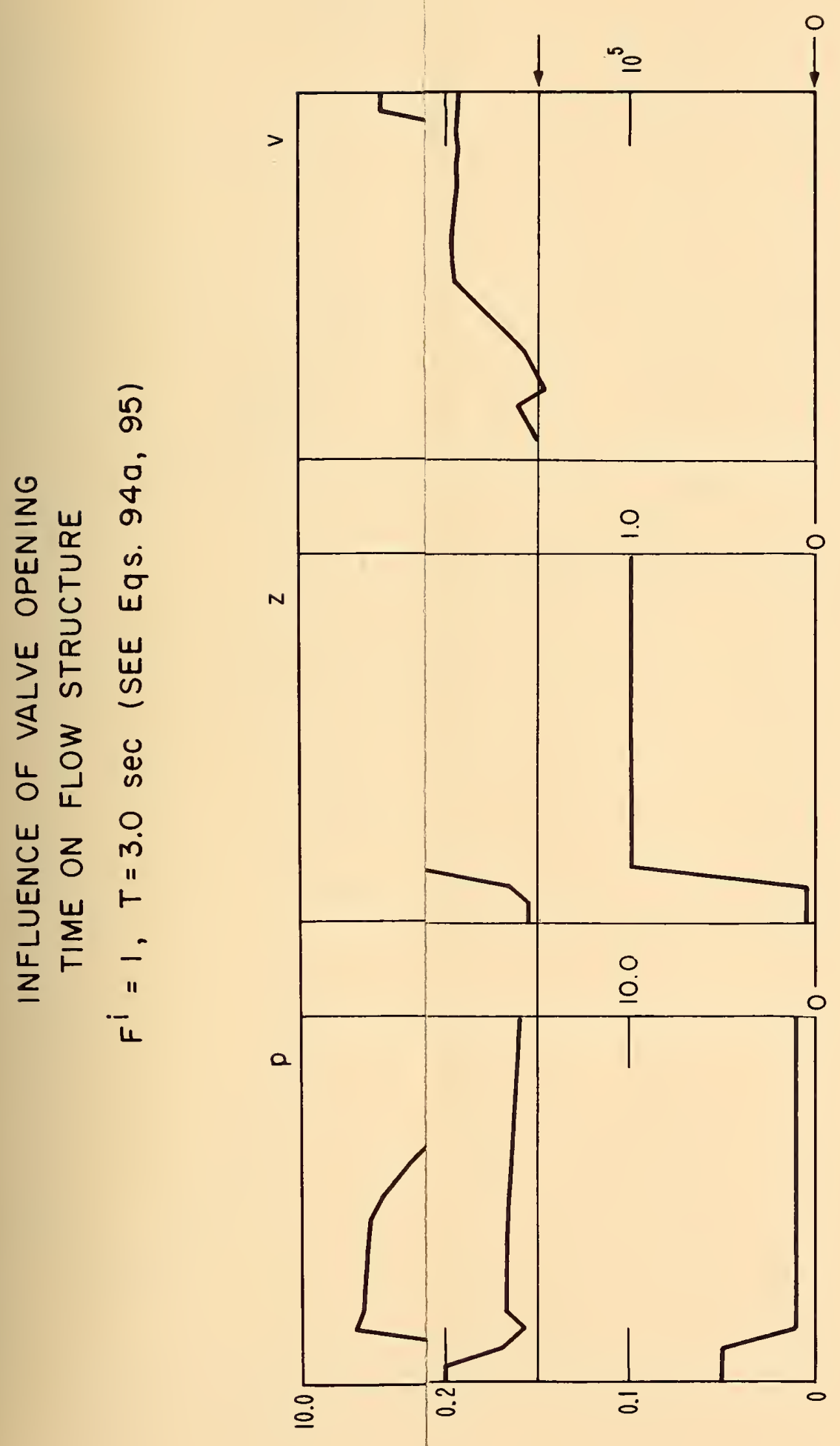

$n$
0
$\frac{0}{3}$
$\frac{0}{4}$

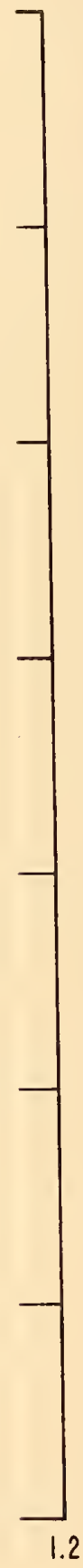





$$
\begin{aligned}
& \text { INFLUENCE OF VALVE OPENING } \\
& \text { TIME ON FLOW STRUCTURE } \\
& F^{i}=1, T=3.0 \mathrm{seC} \text { (SEE EqS. 940,95) }
\end{aligned}
$$

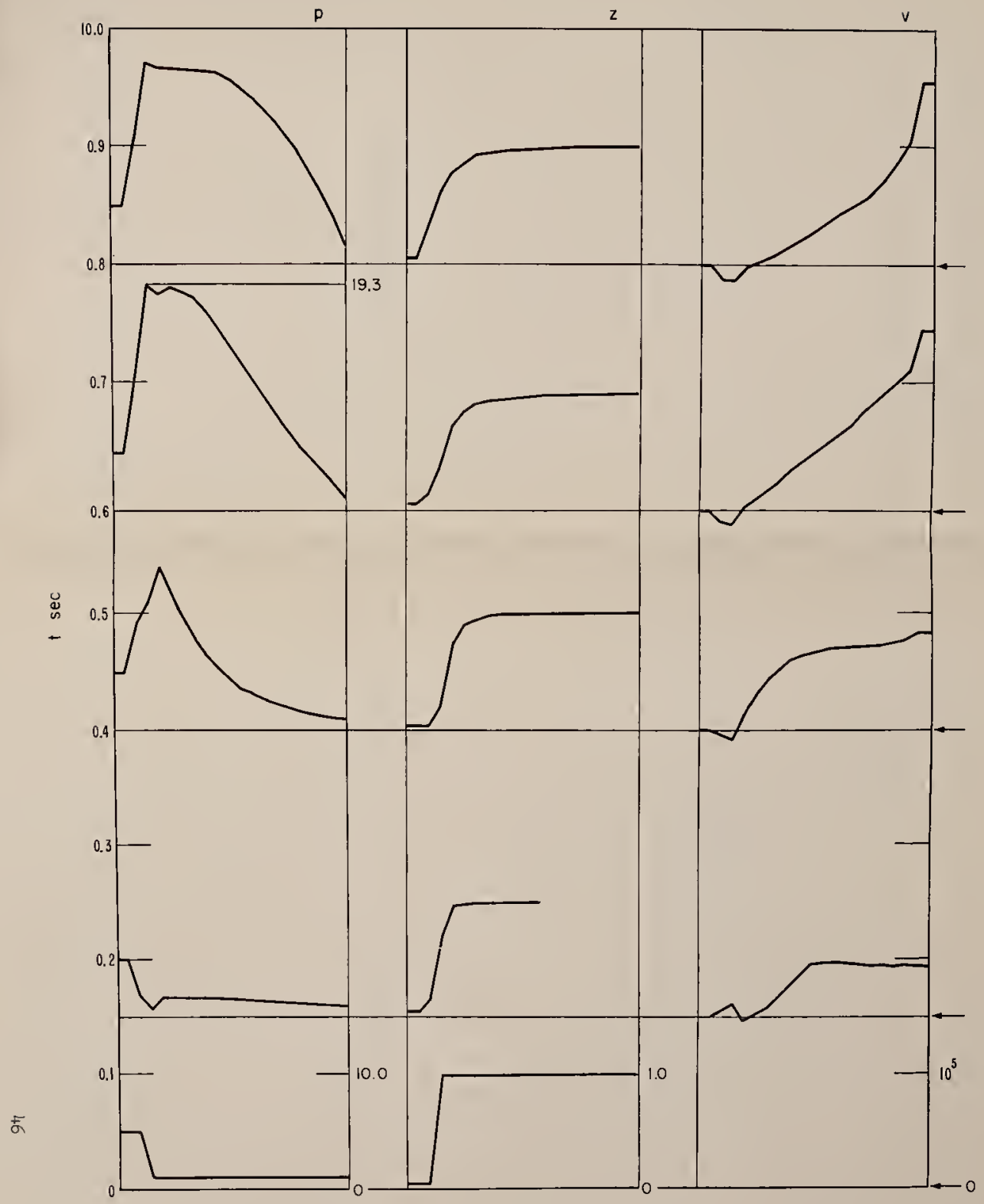

Figure 5 

PRESSURE VERSUS TIME AT A

POINT 20 FEET FROM THE VALVE

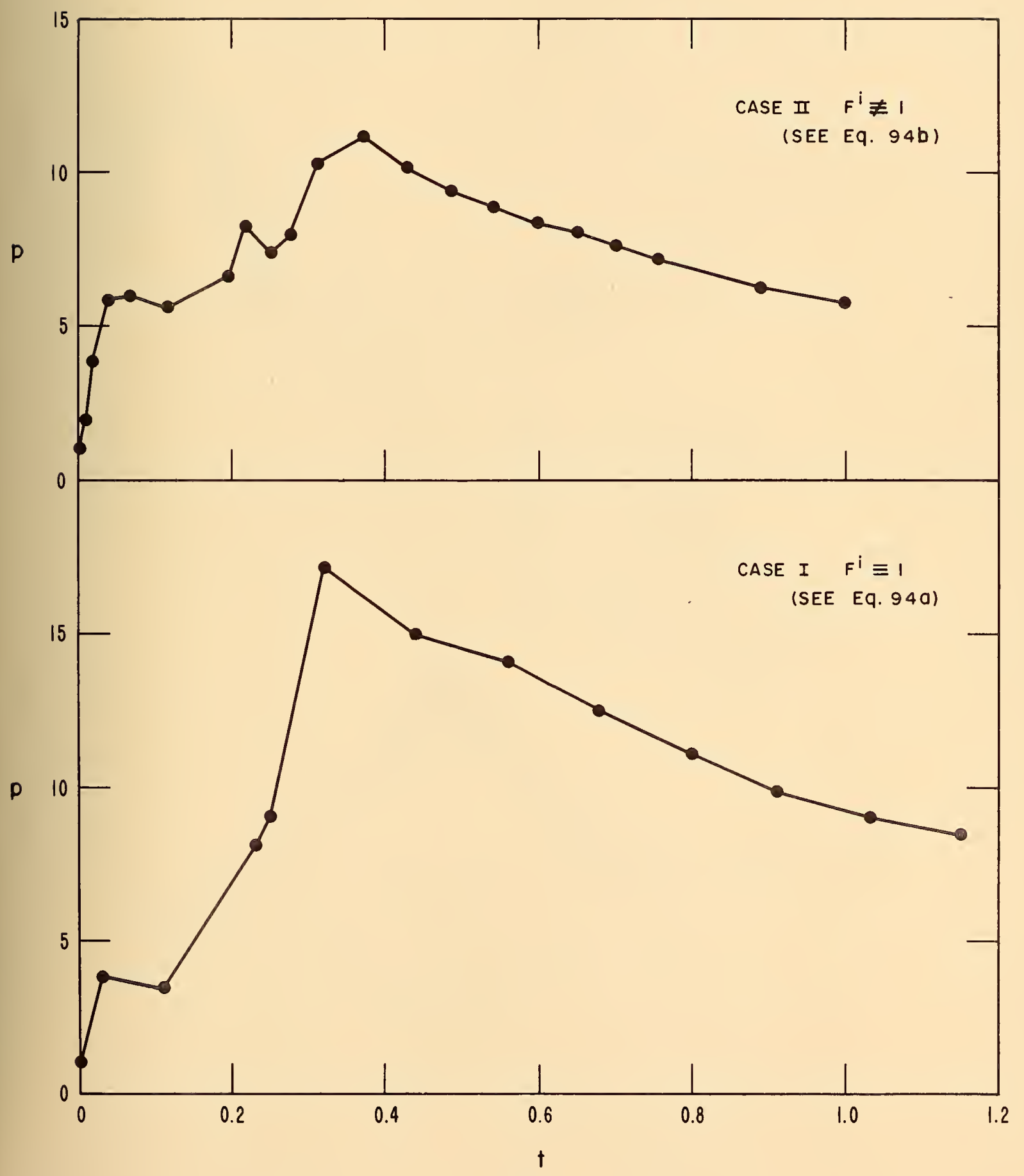

Figure 6 

TYPICAL EXPERIMENTAL CURVES OF PRESSURE AND LIQUID VOLUME FLOW-RATE DURING FIRST SURGE CYCLE FOR ONE DRIVING PRESSURE

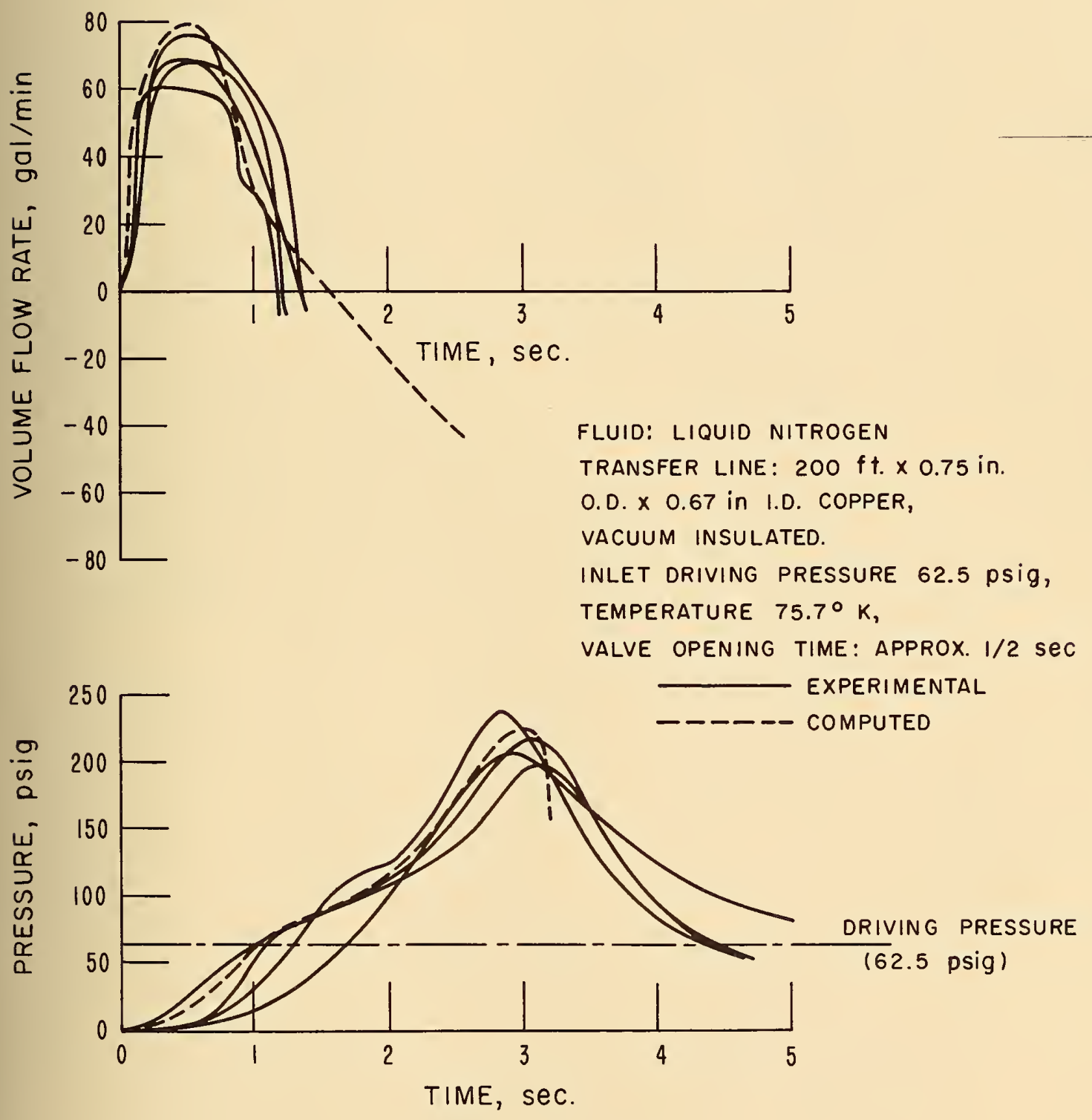

Figure 7 
<smiles>[AlH][In]</smiles> 
(20) 